\title{
Model Predictive Control of NCS with Data Quantization and Bounded Arbitrary Time Delays
}

\author{
Jimin Yu, ${ }^{1,2}$ Yanan Xie, ${ }^{2}$ and Xiaoming Tang \\ ${ }^{1}$ Key Laboratory of Industrial Internet of Things \& Networked Control, Ministry of Education, \\ Chongqing University of Posts and Telecommunications, Chongqing 400065, China \\ ${ }^{2}$ Research Center on Complex System Analysis and Control, Chongqing University of Posts and Telecommunications, \\ Chongqing 400065, China \\ Correspondence should be addressed to Xiaoming Tang; txmmyeye@126.com
}

Received 4 November 2014; Revised 16 March 2015; Accepted 16 March 2015

Academic Editor: Onur Toker

Copyright (C) 2015 Jimin Yu et al. This is an open access article distributed under the Creative Commons Attribution License, which permits unrestricted use, distribution, and reproduction in any medium, provided the original work is properly cited.

\begin{abstract}
The model predictive control for constrained discrete time linear system under network environment is considered. The bounded time delay and data quantization are assumed to coexist in the data transmission link from the sensor to the controller. A novel NCS model is specially established for the model predictive control method, which casts the time delay and data quantization into a unified framework. A stability result of the obtained closed-loop model is presented by applying the Lyapunov method, which plays a key role in synthesizing the model predictive controller. The model predictive controller, which parameterizes the infinite horizon control moves into a single state feedback law, is provided which explicitly considers the satisfaction of input and state constraints. Two numerical examples are given to illustrate the effectiveness of the derived method.
\end{abstract}

\section{Introduction}

Network control system (NCS) is feedback control systems with spatially distributed system components (sensors, actuators, and controllers). As in [1], the information is transformed through a shared band-limited digital communication network, which makes NCS different from other systems. Advantages of NCS include low cost, simple system maintenance, high reliability, and less wiring. The applications of NCS can be building energy efficiency control, intelligent traffic systems, urban sewage treatment, multiple mobile autonomous robots, flight control, satellite attitude control system, and so forth. However, band-limited channels, quantization effects, time delay, and packet dropout are inevitable in NCS, which makes the traditional control theory cannot be directly applied to NCS. Therefore, many researchers are actively exploring the new ways which can effectively process the problem in NCS; the related papers are $[2,3]$.

Obviously, the time delay is an important issue of NCS. The existence of time delay can lead to the deterioration of system performance. To solve this problem, [4] has studied the design of controller of NCS with network-induced time delays which is random at each sampling instant and less than one sampling time. The work in [5] has extended the results of [4] to the case of longer delays. The work in [6] has considered the time varying state delay and the constant time delay. The system is modeled as a Markovian jump model. The work in [7] is to minimize network traffic between a centralized controller and a multivariable plant by using moving horizon techniques. The work in [8] has proposed the model predictive control (MPC) strategy of system with network-induced time delays described by Markovian chains. The work in [9] has used the adaptive predictive functional control to process the discrete state space model with variable time delays. Different from the previous paper, [10] has used a new model and provided a comprehensive approach of MPC for NCS with bounded arbitrary time delay and data packets disorder. Considering all the delay cases, an augmented state space model is obtained. The problem of physical constraints and stability of the system are also considered in the paper. But in [10], the quantization problem is not considered.

Similar to time delay, the existence of data quantization may also result in the deterioration or instability of the system. So far, there have been many papers studied on 
this issue. In [11], the optimal logarithmic quantizer is given which is about single-input-single-output (SISO) discrete linear time-invariant (LTI) system. In [12], the sector bound method is used to transform the quadratic stabilization problem into the robust control problem by generalizing the results in [11]. In [13], the stability of a state feedback modelbased networked control systems (MB-NCS) under uniform quantization schemes is characterized. In [14], a unifying design approach for NCS, quantized control systems (QCS) and their combinations, which is referred to as networked and quantized control systems (NQCS) is viewed. The controller emulation is modeled as sampled-data systems. The work in [15] has discussed the stability of quantized NCS under different initial quantization errors and maximum allowable equivalent delay bound with data packed dropout and obtained a valid model. In [16], the author studied the stabilization of NCS with data quantization and packet dropout and proposed a new model and a novel approach to tackle the issues. The main idea is that the controllers use the previous information to stabilize NCS when packet dropout occurs. The treatment for quantization is mainly through sector bound approach in [12].

Model predictive control (MPC) appeared in the 1970s. As in [17-21], so far, it is already widely applied in complex industrial processes. The defining feature is to deal with constrained problem using the receding horizon optimization method. Hence, if we generalize the approach of MPC to the network environment, the problems existing in NCS will be solved effectively. In order to obtain future control inputs, optimization is performed at each sampling time. The first control move is implemented, and the state measurements are used to perform optimization problem. The readers can refer to $[22,23]$, which systematically introduced the method of MPC.

In this paper, a synthesis approach of MPC for NCS which considers bounded arbitrary time delay and data quantization is given by generalizing the literature $[10,12]$. In detail, the model predictive control for constrained discrete time linear system under network environment with bounded time delay and data quantization is considered. A novel NCS model is specially established for the model predictive control method, which casts the time delay and data quantization into a unified framework in the data transmission link from the sensor to the controller. The satisfaction of input and state constraints are explicitly considered during the construction of the model predictive controller which parameterizes the infinite horizon control moves into a single state feedback law.

Notation. I is the identity matrix with appropriate dimension. The symbol $(*)$ means the matrix is symmetrical. $x(k+i \mid k)$ is the state value at $k+i$, which is predicted by $k$.

\section{Problem Statement}

The framework of NCS considered in this paper is depicted in Figure 1. The plant is a linear time-invariant (LTI) system:

$$
x(k+1)=A x(k)+B u(k), \quad k \geq 0,
$$

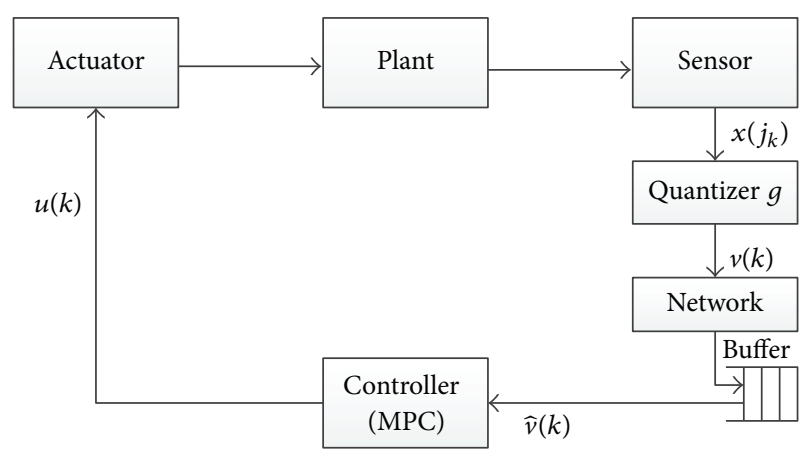

FIGURE 1: Structure of NCS.

where $A$ and $B$ are constant matrices of appropriate dimensions, $k \geq 0$ is the time step, and $u(k) \in R^{m}$ and $x(k) \in R^{n}$ are input and measurable state, respectively. $v(k)$ is the output of quantizer $g$ and $\widehat{v}(k)$ is the input of the controller.

The assumptions of the NCS are as follows.

(A1) The sensor, the controller, and the actuator are time-synchronized.

(A2) The sensor is clock-driven; it sends $x(k)$ at each $k$. The controller is event-driven; it calculates $u$ just when receiving a new $\widehat{v}$. The actuator is event-driven; it updates $u$ when receiving a new $u$.

(A3) In the S-C link, a single data packet $x(k)$ is marked by time stamp and is sent, which is subject to quantization and possibility of time delay, at each sampling time $k$.

(A4) The buffer is supposed to be large enough to store the data which arrived. According to the rule of last-in-first-out, the controller only uses the newest arrived data to calculate the control move.

At each sampling time, a single data packet $x(k)$ is sent by the sensor. Due to the impact of quantization, the value of the data packet may or may not be changed. As the existence of time delays, the data packet will arrive at the buffer on time or arrive in the future time. If the buffer receives new data, it will send the signal to the controller. Then the controller will calculate the new control move $u$; otherwise it keeps the original value.

2.1. Quantizer Description. According to (A3), the $x$ is quantized before it is sent to the network. The quantization process is modeled as

$$
v(k)=g(x(k)), \quad k \geq 0,
$$

where $g(x(k))=\left[g\left(x_{1}(k)\right), g\left(x_{2}(k)\right), \ldots, g\left(x_{n}(k)\right)\right]$ and $g(\cdot)$ is a logarithmic quantizer which is supposed to satisfy $g(-x)=$ $-g(x)$.

A logarithmic quantizer satisfies

$$
\begin{array}{r}
U=\left\{ \pm u_{i}: u_{i}=\rho_{i} u_{0}, i= \pm 1, \pm 2, \ldots\right\} \cup\left\{ \pm u_{0}\right\} \cup\{0\}, \\
0<\rho<1, \quad u_{0}>0,
\end{array}
$$




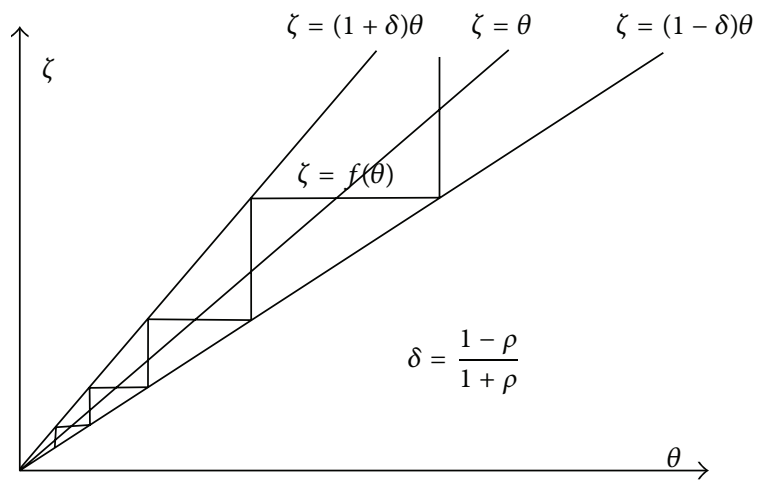

FIGURE 2: Logarithmic quantizer.

where $U$ is the set of quantized levels. Each segment $\left(V_{i}\right)$ of the quantizer is mapped to the corresponding quantization level.

The associated quantizer $g$ is defined as follows:

$$
\zeta(\theta)= \begin{cases}\zeta_{s}, & \text { if } \frac{1}{1+\delta} \zeta_{s}<\theta \leq \frac{1}{1-\delta} \zeta_{s}, \quad \theta>0, \\ 0, & \text { if } \theta=0, \\ -\zeta(-\theta), & \text { if } \theta<0,\end{cases}
$$

where $\zeta$ is $g$ and $\delta$ is a known number which satisfies

$$
\delta=\frac{1-\rho}{1+\rho} .
$$

The logarithmic quantizer is depicted in Figure 2; the sector bound of the logarithmic quantizer is just related to $\delta$.

Remark 1. In contrast, the nonlogarithmic quantizer is depicted in Figure 3. It needs two parameters $\delta^{-}$and $\delta^{+}$to describe the sector bound. It should have a default output value $\zeta_{0}$; if $\zeta_{0}=0$, then $\delta^{-}=-1$; otherwise, $\delta^{+}=\infty$.

According to the sector bound approach in [12]

$$
g(x(k))=\left(I+\Delta_{g}(k)\right) x(k),
$$

where $\Delta_{g}(k)=\operatorname{diag}\left\{\Delta_{g}^{1}(k), \Delta_{g}^{2}(k), \ldots, \Delta_{g}^{n}(k)\right\}$, with $\left|\Delta_{g}^{j}(k)\right| \leq \delta_{g}, j \in\{1,2, \ldots, n\}$.

Let $\mathscr{G}=\left\{G_{j}: j \in \mathcal{N}:=\left\{1,2, \ldots, 2^{n}\right\}\right\}$ be the set of diagonal matrices whose diagonal elements are either $-\delta_{g}$ or $\delta_{g}$. Then, by denoting by $\operatorname{Co}\{\cdot\}$ the convex hull, it is shown that $\Delta_{g}(k) \in \Omega^{g}=\operatorname{Co}\left\{G_{j}: j \in \mathcal{N}\right\}$. There exist nonnegative $\beta_{j}(k)$ 's such that

$$
\Delta_{g}(k)=\sum_{j=1}^{2^{n}} \beta_{j}(k) G_{j},
$$

with $\sum_{j=1}^{2^{n}} \beta_{j}(k)=1$.

2.2. Bounded Time Delays Description. Similar to [10], a sequence $\widehat{\mathscr{J}}=\left\{\hat{\jmath}_{1}, \hat{\jmath}_{2}, \widehat{\jmath}_{3}, \ldots\right\}$ is introduced to describe the

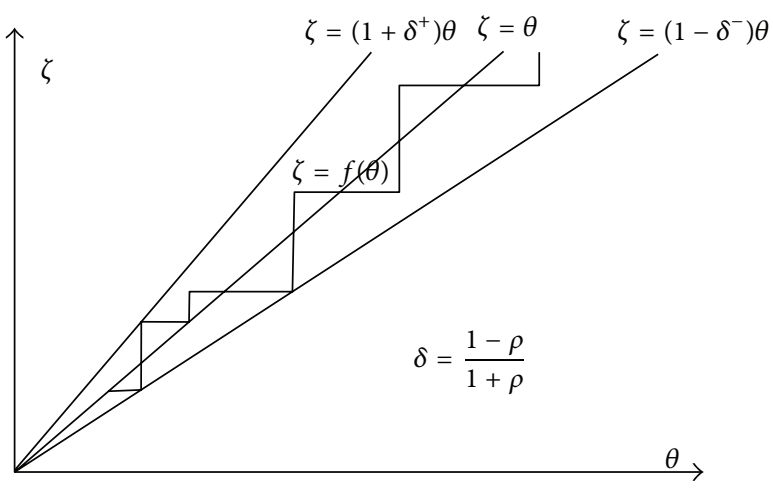

FIgURE 3: Nonlogarithmic quantizer.

time points that quantized data $v(k)$ arriving at the buffer. In the case of data packets disorder, it is not necessary that $\widehat{\jmath}_{1} \leq \hat{\jmath}_{2} \leq \hat{\jmath}_{3} \leq \cdots$. The following ordering operator ord $\{\cdot\}$ is given to process the data packets disorder.

Definition 2. The ordering operator ord $\{\cdot\}[24,25]$ is reordering for the sequence. For any sequence $\left\{e_{1}, e_{2}, \ldots, e_{r}\right\}$, $\operatorname{ord}\left\{e_{1}, e_{2}, \ldots, e_{r}\right\}=\left\{\bar{e}_{1}, \bar{e}_{2}, \ldots, \bar{e}_{r}\right\}$, where $\bar{e}_{m}=e_{n}$, $m, n \in\{1,2, \ldots, r\}$. One obtained the ordered sequence $\left\{\bar{e}_{1}, \bar{e}_{2}, \ldots, \bar{e}_{r}\right\}$ by rearranging $\left\{e_{1}, e_{2}, \ldots, e_{r}\right\}$ without removing or adding any element.

Then we obtain a new ordered sequence $\overline{\mathscr{J}}=$ $\left\{\bar{\jmath}_{1}, \bar{\jmath}_{2}, \bar{\jmath}_{3}, \ldots\right\}$, where $\overline{\mathscr{J}}=\operatorname{ord}\{\widehat{\mathscr{J}}\}$. During one sampling interval, it may happen that more than one packet arrives at the buffer, but only the newest arrived data is utilized and the others are discarded. Hence, only a part of $\overline{\mathscr{J}}$ affects the system. By deleting the discarded elements, we can get the sequence $\mathscr{J}=\left\{j_{1}, j_{2}, j_{3}, \ldots\right\}$ that affects the controller.

Let $d=\max _{j_{i} \in \mathcal{F}}\left(j_{i+1}-j_{i}\right)$. Then $d-1$ is the maximum time delay upper bound from the sensor to the controller. $d=1$ means no time delay. We define

$$
\eta\left(j_{i}\right)=j_{i+1}-j_{i}, \quad j_{i} \in \mathscr{J},
$$

where $\eta\left(j_{i}\right)-1$ is the bounded time delay and $\eta\left(j_{i}\right)$ takes values from $\mathscr{D}=\{1,2, \ldots, d\}$ arbitrarily.

In Figure $1, \widehat{v}\left(j_{i}\right)$ is the received data by the controller. We have

$$
\widehat{v}\left(j_{i}\right)= \begin{cases}v\left(j_{i}\right)=g\left(x\left(j_{i}\right)\right), & j_{i}-k_{i}=0, \\ v\left(j_{i}-1\right)=g\left(x\left(j_{i}-1\right)\right), & j_{i}-k_{i}=1, \\ \vdots & \vdots \\ v\left(j_{i}-d+1\right) & \\ =g\left(x\left(j_{i}-d+1\right)\right), & j_{i}-k_{i}=d-1 .\end{cases}
$$

Note that the quantized state $v(k)$ arrived at the buffer at time $k_{i}$, and for time point $j_{i}$, we can always find a corresponding time $\widehat{\jmath}_{k_{i}} \in \widehat{\mathscr{J}}$, such that $j_{i}=\widehat{\jmath}_{k_{i}}$. 


\section{Modeling of NCS with Delays and \\ Data Quantization}

Assume that the networked controller is a state feedback controller $u=K \hat{v}$, where $K$ is to be designed. For $j_{i} \leq k<$ $j_{i+1}$, the control move $u=K \widehat{v}\left(j_{i}\right)$ is sent to the actuator and implemented. Hence, the closed-loop system becomes

$$
x(k+1)=A x(k)+B K \hat{v}\left(j_{i}\right),
$$

where $j_{i} \leq k<j_{i+1}, j_{i} \in \mathcal{J}$.

The closed-loop system at time instants of successful receipts can be written as

$$
x\left(j_{i+1}\right)=A^{\eta\left(j_{i}\right)} x\left(j_{i}\right)+B_{\eta\left(j_{i}\right)} K \widehat{v}\left(j_{i}\right),
$$

where $B_{\eta\left(j_{i}\right)}=\left(A^{\eta\left(j_{i}\right)-1}+A^{\eta\left(j_{i}\right)-2}+\cdots+I\right) B, j_{i} \in \mathscr{J}, \eta\left(j_{i}\right) \in \mathscr{D}$. Equation (11) is equivalent to the following systems:

$$
\begin{aligned}
& \eta\left(j_{i}\right)=1: \\
& x\left(j_{i+1}\right)=A x\left(j_{i}\right)+B_{1} K\left(I+\sum_{j=1}^{2^{n}} \beta_{j}\left(j_{i}\right) G_{j}\right) x\left(j_{i}\right), \\
& x\left(j_{i+1}\right)=A x\left(j_{i}\right) \\
& +B_{1} K\left(I+\sum_{j=1}^{2^{n}} \beta_{j}\left(j_{i}-1\right) G_{j}\right) x\left(j_{i}-1\right), \\
& x\left(j_{i+1}\right)=A x\left(j_{i}\right) \\
& +B_{1} K\left(I+\sum_{j=1}^{2^{n}} \beta_{j}\left(j_{i}-d+1\right) G_{j}\right) x\left(j_{i}-d+1\right), \\
& \eta\left(j_{i}\right)=2: \\
& x\left(j_{i+1}\right)=A^{2} x\left(j_{i}\right)+B_{2} K\left(I+\sum_{j=1}^{2^{n}} \beta_{j}\left(j_{i}\right) G_{j}\right) x\left(j_{i}\right), \\
& x\left(j_{i+1}\right)=A^{2} x\left(j_{i}\right) \\
& +B_{2} K\left(I+\sum_{j=1}^{2^{n}} \beta_{j}\left(j_{i}-1\right) G_{j}\right) x\left(j_{i}-1\right),
\end{aligned}
$$$$
\begin{aligned}
x\left(j_{i+1}\right)= & A^{2} x\left(j_{i}\right) \\
& +B_{2} K\left(I+\sum_{j=1}^{2^{n}} \beta_{j}\left(j_{i}-d+1\right) G_{j}\right) x\left(j_{i}-d+1\right),
\end{aligned}
$$$$
\eta\left(j_{i}\right)=d:
$$$$
x\left(j_{i+1}\right)=A^{d} x\left(j_{i}\right)+B_{d} K\left(I+\sum_{j=1}^{2^{n}} \beta_{j}\left(j_{i}\right) G_{j}\right) x\left(j_{i}\right),
$$

$$
\begin{gathered}
x\left(j_{i+1}\right)=A^{d} x\left(j_{i}\right) \\
+B_{d} K\left(I+\sum_{j=1}^{2^{n}} \beta_{j}\left(j_{i}-1\right) G_{j}\right) x\left(j_{i}-1\right), \\
\vdots \\
x\left(j_{i+1}\right)=A^{d} x\left(j_{i}\right) \\
+B_{d} K\left(I+\sum_{j=1}^{2^{n}} \beta_{j}\left(j_{i}-d+1\right) G_{j}\right) x\left(j_{i}-d+1\right) .
\end{gathered}
$$

There are $d \times d$ different possible systems in (12). Let us choose the augmented state

$$
\begin{aligned}
& z\left(j_{i}\right) \\
& =\left[x\left(j_{i}\right)^{T}, x\left(j_{i}-1\right)^{T}, x\left(j_{i}-2\right)^{T}, \ldots, x\left(j_{i}-d+1\right)^{T}\right]^{T} .
\end{aligned}
$$

Based on (7) and (12), the closed-loop system can be rewritten as

$$
\begin{gathered}
z\left(j_{i+1}\right)=\Phi\left(j_{i}\right) z\left(j_{i}\right), \\
\Phi\left(j_{i}\right)=\sum_{l=1}^{d} \sum_{m=1}^{d} \omega_{l}\left(j_{i}\right) \lambda_{m}\left(j_{i}\right) \Phi_{l m}, \\
\omega_{l}\left(j_{i}\right)= \begin{cases}1, & j_{i+1}-j_{i}=l, \\
0, & \text { otherwise },\end{cases} \\
\lambda_{m}\left(j_{i}\right)= \begin{cases}1, & j_{i}-k_{i}+1=m, \\
0, & \text { otherwise },\end{cases} \\
\Phi_{l m}=\sum_{j=1}^{2^{n}} \beta_{j}(k) \Phi_{l m j},
\end{gathered}
$$

where $j_{i}=\widehat{\jmath}_{k_{i}}, \Phi_{l m j} \in R^{(d n) \times(d n)}, \Phi_{l m j}=\left[\begin{array}{c}\phi_{l m j} \\ {\left[\begin{array}{ll}I & 0\end{array}\right]}\end{array}\right], \phi_{l m j} \epsilon$ $R^{(l n) \times(d n)}, \phi_{l m j}=\left[\phi_{l m j}^{1}, \phi_{l m j}^{2}, \ldots, \phi_{l m j}^{d}\right], \phi_{l m j}^{s} \in R^{(l n) \times(n)}, s \in$ $\{1,2, \ldots, d\} ; \phi_{l 1 j}^{s}=0, s \neq 1$, and

$$
\phi_{l 1 j}^{1}=\left[\begin{array}{c}
A^{l}+B_{l}\left(K+K G_{j}\right) \\
A^{l-1}+B_{l-1}\left(K+K G_{j}\right) \\
\vdots \\
A^{\max \{1, l-d\}}+B_{\max \{1, l-d\}}\left(K+K G_{j}\right)
\end{array}\right] \text {, }
$$


for $m \neq 1, \phi_{\text {lmj }}^{s}=0, s \neq\{1, m\}$, and

$$
\left[\begin{array}{ll}
\phi_{l m j}^{1} & \phi_{l m j}^{m}
\end{array}\right]=\left[\begin{array}{cc}
A^{l} & B_{l}\left(K+K G_{j}\right) \\
A^{l-1} & B_{l-1}\left(K+K G_{j}\right) \\
\vdots & \vdots \\
A^{\max \{1, l-d\}} & B_{\max \{1, l-d\}}\left(K+K G_{j}\right)
\end{array}\right] .
$$

Lemma 3. Consider system (1), where both data quantization and time delay may occur. By applying state feedback controller defined by $u=K \hat{v}$, the closed-loop system (14) is asymptotically stable if there exists a positive-definite matrix $P$ such that

$$
\Phi_{l m j}^{T} P \Phi_{l m j}-P<0, \quad l, m \in \mathscr{D}, j \in \mathcal{N} .
$$

Proof. We define the following Lyapunov function:

$$
V\left(z\left(j_{i}\right)\right)=z\left(j_{i}\right)^{T} P z\left(j_{i}\right) .
$$

By using (14), we have

$$
V\left(z\left(j_{i+1}\right)\right)=z\left(j_{i}\right)^{T} \Phi\left(j_{i}\right)^{T} P \Phi\left(j_{i}\right) z\left(j_{i}\right) .
$$

In order to ensure asymptotic stability of the system, we have

$$
V\left(z\left(j_{i+1}\right)\right)-V\left(z\left(j_{i}\right)\right)<0 .
$$

Then

$$
\begin{aligned}
& V\left(z\left(j_{i+1}\right)\right)-V\left(z\left(j_{i}\right)\right) \\
& =z\left(j_{i}\right)^{T}\left(\Phi\left(j_{i}\right)^{T} P \Phi\left(j_{i}\right)-P\right) z\left(j_{i}\right) \\
& =z\left(j_{i}\right)^{T}\left(\sum_{j=1}^{2^{n}} \beta_{j}(k) \sum_{l=1}^{d} \sum_{m=1}^{d} \omega_{l}\left(j_{i}\right)\right. \\
& \left.\cdot \lambda_{m}\left(j_{i}\right)\left(\Phi_{l m j}^{T} P \Phi_{l m j}-P\right)\right) \\
& \cdot z\left(j_{i}\right) .
\end{aligned}
$$

Then, we obtain (18). The asymptotic stability of (14) is also guaranteed.

\section{Stabilization of NCS via MPC}

In this section, we will introduce the synthesis approach of MPC to process NCS with data quantization and time delay. Also, the input and state constraints are considered, which can be expressed as follows:

$$
\begin{gathered}
-\underline{u} \leq u\left(j_{1}+i\right) \leq \bar{u}, \\
-\underline{\psi} \leq \Psi x\left(j_{1}+i+1\right) \leq \bar{\psi}, \quad \forall i \geq 0,
\end{gathered}
$$

where $\underline{u}=\left[\underline{u}_{1}, \underline{u}_{2}, \ldots, \underline{u}_{m}\right]^{T}, \bar{u}=\left[\bar{u}_{1}, \bar{u}_{2}, \ldots, \bar{u}_{m}\right]^{T}, \underline{u}_{j}>0$, $\bar{u}_{j}>0, j \in\{1,2, \ldots, m\} ; \underline{\psi}=\left[\underline{\psi}_{1}, \underline{\psi}_{2}, \ldots, \underline{\psi}_{m}\right]^{T}, \bar{\psi}=$ $\left[\bar{\psi}_{1}, \bar{\psi}_{2}, \ldots, \bar{\psi}_{m}\right]^{T}, \underline{\psi}_{s}>0, \bar{\psi}_{s}>0, s \in\{1,2, \ldots, q\} ; \Psi \in R^{q \times n}$. below.
Lemma 4 (Schur complements). If $C>0$, then

$$
\left[\begin{array}{cc}
C & D \\
D^{T} & E
\end{array}\right] \geq 0 \Longleftrightarrow E \geq 0, \quad E-D^{T} C^{-1} D \geq 0,
$$

and if $E>0$, then

$$
\left[\begin{array}{cc}
C & D \\
D^{T} & E
\end{array}\right] \geq 0 \Longleftrightarrow C \geq 0, \quad C-D E^{-1} D^{T} \geq 0
$$

where $C=C^{T}$ and $E=E^{T}$.

4.1. Optimization Problem for MPC. At $j_{i}$, an MPC optimization problem is solved and the obtained control move is implemented for $j_{i} \leq k<j_{i+1}$. At the next successfully transformed sampling time $j_{i+1}$, the same optimization problem will be computed with renewed receipt. For the purpose of driving (1) to the equilibrium point, the control move $u(k)=$ $u\left(j_{i}\right)=F\left(j_{i}\right) \widehat{v}\left(j_{i}\right)$ will be utilized.

Taking (11) as the predictive model, we can obtain the closed-loop state predictions

$$
\begin{aligned}
x\left(j_{i+h+1} \mid j_{i}\right)= & A^{\eta\left(j_{i+h}\right)} x\left(j_{i+h} \mid j_{i}\right) \\
& +B_{\eta\left(j_{i+h}\right)} F\left(j_{i}\right) \hat{v}\left(j_{i+h} \mid j_{i}\right),
\end{aligned}
$$

where $h \geq 0, \eta\left(j_{i+h}\right) \in \mathscr{D}$, and

$$
x\left(j_{i} \mid j_{i}\right)=\sigma\left(j_{i}\right) v\left(j_{i} \mid j_{i}\right)=\sigma\left(j_{i}\right) \widehat{v}\left(j_{i}\right)
$$

where $\sigma\left(j_{i}\right)=\operatorname{diag}\left\{\sigma^{1}\left(j_{i}\right), \sigma^{2}\left(j_{i}\right), \ldots, \sigma^{n}\left(j_{i}\right)\right\},\left(1+\delta_{g}\right)^{-1} \leq$ $\sigma^{i}\left(j_{i}\right) \leq\left(1-\delta_{g}\right)^{-1}, i \in\{1,2, \ldots, \eta\}$. Let $\mathscr{C}=\left\{C_{j}: j \in \mathscr{N}\right\}$ be the set of diagonal matrices whose diagonal elements are either $\left(1-\delta_{g}\right)^{-1}$ or $\left(1-\delta_{g}\right)^{-1}$. Then, it can be seen that $\sigma\left(j_{i}\right) \in \Omega^{\sigma}=\operatorname{Co}\left\{C_{j}: j \in \mathcal{N}\right\}$.

Remark 5. It should be emphasized that the closed-loop model in (26) is generalized from the closed-loop model in [10]. If $\widehat{v}\left(j_{i+h} \mid j_{i}\right)=g\left(\widehat{x}\left(j_{i+h} \mid j_{i}\right)\right)=\widehat{x}\left(j_{i+h} \mid j_{i}\right)$, then (26) is reduced to

$$
\begin{aligned}
& x\left(j_{i+h+1} \mid j_{i}\right)= A^{\eta\left(j_{i+h}\right)} x\left(j_{i+h} \mid j_{i}\right) \\
&+B_{\eta\left(j_{i+h}\right)} F\left(j_{i}\right) \hat{x}\left(j_{i+h} \mid j_{i}\right), \\
& h \geq 0, \quad \eta\left(j_{i+h}\right) \in \mathscr{D} .
\end{aligned}
$$

Obviously, the expression of control input is the major difference between the model in (26) and the model in [10]. In (26), the control move $u$ is $u\left(j_{i}\right)=F\left(j_{i}\right) \widehat{v}\left(j_{i}\right)$, which is not the same as $u\left(j_{i}\right)=F\left(j_{i}\right) \hat{x}\left(j_{i}\right)$ in [10]. As the existence of this difference, the stabilization results in this paper are different from the results in [10], and these different results are the major contribution of this paper. 
The MPC algorithm considered in this paper can be expressed as the following min-max optimization problem:

$$
\begin{array}{ll}
\min _{F\left(j_{i}\right)} \max _{\eta\left(j_{i}\right) \in \mathscr{D}} & J\left(j_{i}\right) \\
\text { s.t. } & -\underline{\psi} \leq \Psi x\left(j_{i+h+1} \mid j_{i}\right) \leq \bar{\psi} \\
& -\underline{u} \leq u\left(j_{i+h} \mid j_{i}\right) \leq \bar{u}, \quad h \geq 0 \\
& (26),(27),
\end{array}
$$

where $J\left(j_{i}\right)=\sum_{h=0}^{\infty}\left[\left\|x\left(j_{i+h} \mid j_{i}\right)\right\|_{W}^{2}+\left\|u\left(j_{i+h} \mid j_{i}\right)\right\|_{R}^{2}\right] . R>0$ and $W>0$ are symmetric weighting matrices.

4.2. Stability Condition and Constraints Handling. In order to derive an upper bound on $\max _{\eta\left(j_{i}\right) \in \mathscr{D}} J\left(j_{i}\right)$ and solve (29)-(32), we impose the stability constraint

$$
\begin{aligned}
& V\left(z\left(j_{i+h+1} \mid j_{i}\right)\right)-V\left(z\left(j_{i+h} \mid j_{i}\right)\right) \\
& \quad \leq-\left\|x\left(j_{i+h} \mid j_{i}\right)\right\|_{W}^{2}-\left\|u\left(j_{i+h} \mid j_{i}\right)\right\|_{R}^{2}, \quad \forall h \geq 0 .
\end{aligned}
$$

For stable closed-loop system, we have $x\left(j_{\infty} \mid j_{i}\right)=0, u\left(j_{\infty} \mid\right.$ $\left.j_{i}\right)=0$, and $V\left(z\left(j_{\infty} \mid j_{i}\right)\right)=0$. By summing (33) from $h=0$ to $h=\infty$, we can obtain

$$
J\left(j_{i}\right) \leq V\left(z\left(j_{i} \mid j_{i}\right)\right) .
$$

Thus

$$
\max _{\eta\left(j_{i}\right) \in \mathscr{D}} J\left(j_{i}\right) \leq V\left(z\left(j_{i} \mid j_{i}\right)\right) .
$$

This inequality gives the upper bound of $\max J\left(j_{i}\right)$.

The objective function of the networked MPC algorithm has been redefined. We define a scalar $\gamma$ satisfying $V\left(z\left(j_{i}\right)\right.$ $\left.\left.j_{i}\right)\right) \leq \gamma \cdot Q$ and $P$ are symmetric positive-definite weighting matrices which satisfy $Q=\gamma P^{-1}>0$. Based on Lemma 4, $V\left(z\left(j_{i} \mid j_{i}\right)\right) \leq \gamma$ can be guaranteed by

$$
\left[\begin{array}{cc}
1 & * \\
z\left(j_{i} \mid j_{i}\right) & Q
\end{array}\right] \geq 0
$$

In (36), $z\left(j_{i} \mid j_{i}\right)=\left[\left(\sigma\left(j_{i}\right) v\left(j_{i} \mid j_{i}\right)\right)^{T}, x\left(j_{i}-1 \mid j_{i}\right)^{T}, \ldots, x\left(j_{i}-\right.\right.$ $\left.\left.d+1 \mid j_{i}\right)^{T}\right]^{T}$. For calculating $z\left(j_{i} \mid j_{i}\right)$, we should calculate every element of the vector $[10,16] . x\left(j_{i}-1 \mid j_{i}\right), x\left(j_{i}-2 \mid\right.$ $\left.j_{i}\right), \ldots, x\left(j_{i}-d+1 \mid j_{i}\right)$ can be obtained by the following iterative method:

$$
\begin{gathered}
x\left(j_{i}-1 \mid j_{i}\right)=A^{-1} \sigma\left(j_{i}\right) v\left(j_{i} \mid j_{i}\right)-A^{-1} B F\left(j_{i}\right) \hat{v}\left(j_{i}-1\right), \\
x\left(j_{i}-2 \mid j_{i}\right)=A^{-2} \sigma\left(j_{i}\right) v\left(j_{i} \mid j_{i}\right)-A^{-2} B F\left(j_{i}\right) \hat{v}\left(j_{i}-1\right) \\
-A^{-1} B F\left(j_{i}\right) \widehat{v}\left(j_{i}-2\right), \\
\vdots \\
x\left(j_{i}-d+1 \mid j_{i}\right)=A^{-d+1} \sigma\left(j_{i}\right) v\left(j_{i} \mid j_{i}\right) \\
-\sum_{N=1}^{d-1} A^{-N} B F\left(j_{i}\right) \hat{v}\left(j_{i}-d+N\right) .
\end{gathered}
$$

Apparently, $\left[u\left(j_{i}-1\right), u\left(j_{i}-2\right), \ldots, u\left(j_{i}-d+1\right)\right]=\left[F\left(j_{i}\right) \widehat{v}\left(j_{i}-\right.\right.$ $\left.1), F\left(j_{i}\right) \widehat{v}\left(j_{i}-2\right), \ldots, F\left(j_{i}\right) \widehat{v}\left(j_{i}-d+1\right)\right]$ should be stored by the controller at each $j_{i}$. Note that the calculation method of $u$ is different from [10].

In order to update $z\left(j_{i} \mid j_{i}\right)$, at each $j_{i}$, the controller should store $x\left(j_{i} \mid j_{i}\right)$ and $u\left(j_{i}-1\right), u\left(j_{i}-2\right), \ldots, u\left(j_{i}-d+1\right)$ for future usage. In (33), $u\left(j_{i+h} \mid j_{i}\right)=F\left(j_{i}\right) \Xi\left(j_{i+h} \mid j_{i}\right) g\left(z\left(j_{i+h} \mid\right.\right.$ $\left.\left.j_{i}\right)\right)$, where $\Xi\left(j_{i+h} \mid j_{i}\right)$ satisfies $\widehat{v}\left(j_{i+h} \mid j_{i}\right)=\Xi\left(j_{i+h} \mid j_{i}\right) \times$ $g\left(z\left(j_{i+h} \mid j_{i}\right)\right)$. Denote $\Xi_{m}=\Xi\left(j_{i+h} \mid j_{i}\right)=\left[0, \ldots, I_{m}, 0, \ldots, 0\right]$ (all blocks are zeros except the $m$ th block), where $j_{i+h}-k_{i+h}+$ $1=m$. For all $z\left(j_{i+h} \mid j_{i}\right),(33)$ is satisfied if and only if

$$
\begin{aligned}
\Pi\left(j_{i+h} \mid j_{i}\right)= & \Phi\left(j_{i+h} \mid j_{i}\right)^{T} P \Phi\left(j_{i+h} \mid j_{i}\right)-P \\
& +[I, 0, \ldots, 0]^{T} W[I, 0, \ldots, 0] \\
& +\Xi\left(j_{i+h} \mid j_{i}\right)^{T}\left(I+\Delta_{g}\left(j_{i}\right)\right)^{T} \\
& \cdot R\left(I+\Delta_{g}\left(j_{i}\right)\right) \Xi\left(j_{i+h} \mid j_{i}\right) \leq 0,
\end{aligned}
$$

where $\Phi\left(j_{i+h} \mid j_{i}\right)$ is the same as $\Phi\left(j_{i} \mid j_{i}\right)$ in (14), except that $K$ is replaced by $F\left(j_{i}\right)$. According to the augmented state space model (14), we have

$$
\Pi\left(j_{i+h} \mid j_{i}\right)=\sum_{l=1}^{d} \sum_{m=1}^{d} \omega_{l}\left(j_{i+h}\right) \lambda_{m}\left(j_{i+h}\right) \sum_{j=1}^{2^{n}} \beta_{j}\left(j_{i}\right) \Pi_{l m j},
$$

where $\Pi_{l m j}=\Phi_{l m j}^{T} P \Phi_{l m j}-P+[I, 0, \ldots, 0]^{T} W[I, 0, \ldots, 0]+$ $\Xi_{m}^{T}\left(F\left(j_{i}\right)\left(I+G_{j}\right)\right)^{T} R \times\left(F\left(j_{i}\right)\left(I+G_{j}\right)\right) \Xi_{m}$ and

$$
\begin{gathered}
\omega_{l}\left(j_{i+h}\right)= \begin{cases}1, & j_{i+h+1}-j_{i+h}=l, \\
0, & \text { otherwise },\end{cases} \\
\lambda_{m}\left(j_{i+h}\right)= \begin{cases}1, & j_{i+h}-k_{i+h}+1=m, \\
0, & \text { otherwise. }\end{cases}
\end{gathered}
$$

Similar to Lemma 3, (38) is equivalent to

$$
\begin{aligned}
\Phi_{l m j}^{T} P \Phi_{l m j}-P \leq & -[I, 0, \ldots, 0]^{T} W[I, 0, \ldots, 0] \\
& -\Xi_{m}^{T}\left(F\left(j_{i}\right)\left(I+G_{j}\right)\right)^{T} R \\
& \times F\left(j_{i}\right)\left(I+G_{j}\right) \Xi_{m}, \\
& j \in\left\{1,2, \ldots, 2^{n}\right\}, \quad l, m \in \mathscr{D} .
\end{aligned}
$$

Then, we deal with the problem of state constraint (30) and input constraint (31). In [26], there is the concept of invariant ellipsoid of nonnetwork MPC. So, (36) and (41) lead to

$$
z\left(j_{i+h} \mid j_{i}\right)^{T} Q^{-1} z\left(j_{i+h} \mid j_{i}\right) \leq 1, \quad \forall h \geq 0 .
$$

Thus, $\varepsilon=\left\{z \mid z^{T} Q^{-1} z \leq 1\right\}$ is an invariant ellipsoid for predicted states of the closed-loop system. 
The symmetric matrix $Q$ is divided into $d \times d$ blocks, which is of the same dimension; that is,

$$
Q=\left[\begin{array}{cccc}
Q^{1,1} & \left(Q^{2,1}\right)^{T} & \cdots & \left(Q^{d, 1}\right)^{T} \\
Q^{2,1} & Q^{2,2} & \cdots & \left(Q^{d, 2}\right)^{T} \\
\vdots & \vdots & \ddots & \vdots \\
Q^{d, 1} & Q^{d, 2} & \cdots & Q^{d, d}
\end{array}\right]
$$

Lemma 6. Suppose there exist a scalar $\gamma$, symmetric matrices $\Gamma, Z$, and $Q$, and any matrices $F\left(j_{i}\right)$ satisfying (33), (36), and the following inequalities:

$$
\begin{aligned}
& F\left(j_{i}\right)\left(I+G_{j}\right) Q^{m, m}\left(F\left(j_{i}\right)\left(I+G_{j}\right)\right)^{T} \leq \Gamma, \\
& j \in\left\{1,2, \ldots, 2^{n}\right\}, \quad \Gamma_{s s} \leq u_{s, \text { inf }}^{2}, \quad s \in \mathbf{m}, \\
& \Psi\left(A^{l}+B_{l} F\left(j_{i}\right)\left(I+G_{j}\right)\right) \\
& \cdot Q^{1,1}\left(A^{l}+B_{l} F\left(j_{i}\right)\left(I+G_{j}\right)\right)^{T} \Psi^{T} \leq Z, \\
& j \in\left\{1,2, \ldots, 2^{n}\right\}, \quad l \in \mathscr{D}, \\
& \Psi\left[\begin{array}{ll}
A^{l} & B_{l} F\left(j_{i}\right)\left(I+G_{j}\right)
\end{array}\right]\left[\begin{array}{ll}
Q^{1,1} & Q^{1, m} \\
Q^{m, 1} & Q^{m, m}
\end{array}\right] \\
& \text { - }\left[\begin{array}{ll}
A^{l} & B_{l} F\left(j_{i}\right)\left(I+G_{j}\right)
\end{array}\right]^{T} \Psi^{T} \leq Z, \\
& j \in\left\{1,2, \ldots, 2^{n}\right\}, \quad Z_{s s} \leq \psi_{s, \text { inf }}^{2}, \\
& s \in \mathbf{q}, \quad l \in \mathscr{D}, \quad m \in\{2,3, \ldots, d\},
\end{aligned}
$$

where $u_{s, \text { inf }}=\min \left\{\underline{u}_{s}, \bar{u}_{s}\right\}, \psi_{s, \inf }=\min \left\{\psi, \bar{\psi}_{s}\right\}$, and $Z_{s s}\left(\Gamma_{s s}\right)$ is the sth diagonal element of $Z(\Gamma)$.

Proof. The proof process can be obtained by generalizing the proof of Lemma 1 in [10]. For simplicity, we omit it.

4.3. LMI Optimization Problem. We use the LMI technique to process the optimization problem $(29)-(32)$. Define $F\left(j_{i}\right)=$ $Y X^{-1}$ and

$$
X_{l m}=\left[\begin{array}{cccc}
X_{l m}^{1,1} & X_{l m}^{1,2} & \cdots & X_{l m}^{1, d} \\
X_{l m}^{2,1} & X_{l m}^{2,2} & \cdots & X_{l m}^{2, d} \\
\vdots & \vdots & \ddots & \vdots \\
X_{l m}^{d, 1} & X_{l m}^{d, 2} & \cdots & X_{l m}^{d, d}
\end{array}\right], \quad X_{l m}^{m, t}=X, t \in \mathscr{D}
$$

where each block in $X_{l m}$ has the same dimension.

By taking a congruence transformation via $X_{l m}$ on each of the inequalities (41), utilizing the inequality $X_{l m}^{T} Q^{-1} X_{l m} \geq X_{l m}^{T}+X_{l m}-Q$ and Lemma 4, the stability condition (33) or (41) can be guaranteed by the following LMIs:

$$
\begin{aligned}
& {\left[\begin{array}{cccc}
X_{l m}^{T}+X_{l m}-Q & * & * & * \\
\Phi_{l m j} X_{l m} & Q & * & * \\
W^{1 / 2}\left[\begin{array}{llll}
X_{l m}^{1,1} & X_{l m}^{1,2} & \cdots & X_{l m}^{1, d}
\end{array}\right] & 0 & \gamma I & * \\
R^{1 / 2}\left[\begin{array}{lllll}
y_{j} & y_{j} & \cdots & y_{j}
\end{array}\right] & 0 & 0 & \gamma I
\end{array}\right] \geq 0,} \\
& l, m \in \mathscr{D}, \quad j \in \mathcal{N},
\end{aligned}
$$

where $y_{j}=Y\left(I+G_{j}\right)$. Notice that in $\Phi_{l m j} X_{l m}$, each $I+G_{j}$ is multiplied with $F\left(j_{i}\right) X$ and $F\left(j_{i}\right) X\left(I+G_{j}\right)$ should be changed as $y_{j}$.

Lemma 7. Utilizing the inequality $X^{T}\left(Q^{m, m}\right)^{-1} X \geq X^{T}+$ $X-Q^{m, m}$ and applying Lemma 4, supposing that there exist symmetric matrices $\Gamma, Z$, and $Q$, (44), (45), and (46) can be guaranteed by the following LMIs separately:

$$
\begin{aligned}
& {\left[\begin{array}{cc}
X^{T}+X-Q^{m, m} & * \\
y_{j} & \Gamma
\end{array}\right] \geq 0,} \\
& m \in \mathscr{D}, \quad j \in \mathcal{N}, \quad \Gamma_{s s} \leq u_{s, \text { inf }}^{2}, \quad s \in \mathbf{m}, \\
& {\left[\begin{array}{cc}
X^{T}+X-Q^{1,1} & * \\
\Psi\left(A^{l} X+B_{l} y_{j}\right) & Z
\end{array}\right] \geq 0,} \\
& l \in \mathscr{D}, \quad j \in \mathcal{N}, \quad Z_{s s} \leq \psi_{s, \text { inf }}^{2}, \quad s \in \mathbf{q}, \\
& {\left[\begin{array}{ccc}
\left(X_{l m}^{1,1}\right)^{T}+X_{l m}^{1,1}-Q^{1,1} & * & * \\
\left(X_{l m}^{1, m}\right)^{T}+X-Q^{m, 1} & X^{T}+X-Q^{m, m} & * \\
\Psi\left(A^{l} X_{l m}^{1,1}+B_{l} y_{j}\right) & \Psi\left(A^{l} X_{l m}^{1, m}+B_{l} y_{j}\right) & Z
\end{array}\right] \geq 0,} \\
& l \in \mathscr{D}, \quad m \in\{2,3, \ldots, d\}, \quad j \in \mathcal{N}, \\
& Z_{s s} \leq \psi_{s, \text { inf }}^{2}, \quad s \in \mathbf{q},
\end{aligned}
$$

where $\Gamma_{s s}\left(Z_{s s}\right)$ is the sth diagonal element of $\Gamma(Z)$.

Thus, the infinite horizon optimization problem (29)(32) is approximately transformed into the following LMI optimization problem:

$$
\min _{\gamma, Q, X_{l m}, Y, Z, \Gamma} \gamma, \quad \text { s.t. (36), (48)-(51). }
$$

For the propose of obtaining the control move $u\left(j_{i}\right)=$ $Y X^{-1} \widehat{v}\left(j_{i}\right)$, the LMI optimization problem (52) is carried out at each $j_{i}$. During the time interval $j_{i} \leq k \leq j_{i+1}$, this control move is implemented by the plant.

Theorem 8. Considering the NCS in Figure $1, u\left(j_{i}\right)=$ $Y X^{-1} g\left(v\left(j_{i}\right)\right), j_{i} \leq k \leq j_{i+1}, i \geq 1$, is obtained by solving the optimization problem (52) at each $j_{i}$. Assume that it is feasible at times $j_{1}$; then it is also feasible for all times $j_{h}, h>1$. Then, the receding horizon sending of the control move $u\left(j_{i}\right)$ 
guarantees the satisfaction of (23). So, the closed-loop system is exponentially stable.

Proof. If the optimization process at $j_{i}$ is also feasible at $j_{i+1}$, then the feasibility of (52) means its feasibility at any $j_{h}, h \geq 1$.

So, we suppose that the optimization process is feasible at $j_{i}$; then we have

$$
z\left(j_{i+h} \mid j_{i}\right)^{T} Q^{-1} z\left(j_{i+h} \mid j_{i}\right) \leq 1, \quad \forall h \geq 0 .
$$

Here, $z\left(j_{i+h} \mid j_{i}\right)=\left[\left(\sigma\left(j_{i}\right) v\left(j_{i+h} \mid j_{i}\right)\right)^{T}, x\left(j_{i+h}-1 \mid j_{i}\right)^{T}, \ldots\right.$, $\left.x\left(j_{i+h}-d+1 \mid j_{i}\right)^{T}\right]^{T}, x\left(j_{i+h}-t+1 \mid j_{i}\right)=A^{-t+1} \sigma\left(j_{i}\right) v\left(j_{i+h} \mid\right.$ $\left.j_{i}\right)-\sum_{N=1}^{t-1} A^{-N} B F\left(j_{i}\right) \widehat{v}\left(j_{i+h}-t+N\right), t \in[1,2, \ldots, d]$, which are not the same as in [10].

Obviously, the satisfying relationship of $z\left(j_{i+1} \mid j_{i}\right)$ applies equally to the case of $z\left(j_{i+1} \mid j_{i+1}\right)$. Hence, we have the following LMI:

$$
\left[\begin{array}{cc}
1 & * \\
z\left(j_{i+1} \mid j_{i+1}\right) & Q
\end{array}\right] \geq 0 .
$$

Due to the state information used by optimization problem (52) is only (36), so the optimization process at $j_{i}$ is also feasible at $j_{i+1}$.

From (33), we have

$$
\begin{aligned}
& V^{*}\left(z^{*}\left(j_{i+1} \mid j_{i}\right)\right)-V^{*}\left(z\left(j_{i} \mid j_{i}\right)\right) \\
& \quad \leq-\left\|\sigma\left(j_{i}\right) v\left(j_{i} \mid j_{i}\right)\right\|_{W}^{2}-\left\|\left(F\left(j_{i}\right) \widehat{v}\left(j_{i}\right)\right)^{*}\right\|_{R}^{2}, \quad \forall h \geq 0 .
\end{aligned}
$$

Here, the $*$ means the optimization process. Define

$$
V^{*}\left(z\left(j_{i} \mid j_{i}\right)\right) \leq \gamma^{*}\left(j_{i}\right)
$$

We have

$$
\begin{aligned}
\gamma\left(j_{i+1}\right)= & \gamma^{*}\left(j_{i}\right)-\max _{m \in \mathscr{D}}\left\|\sigma\left(j_{i}\right) v\left(j_{i} \mid j_{i}\right)\right\|_{W}^{2} \\
& -\left\|\left(F\left(j_{i}\right) \hat{v}\left(j_{i}\right)\right)^{*}\right\|_{R}^{2} .
\end{aligned}
$$

Assume that $\gamma^{*}\left(j_{i+1}\right)$ is the reoptimization of $\gamma\left(j_{i+1}\right)$ at $j_{i+1}$. According to the optimization theory, we have $\gamma^{*}\left(j_{i+1}\right) \leq$ $\gamma\left(j_{i+1}\right)$. So, we have

$$
\begin{gathered}
\gamma^{*}\left(j_{i+1}\right)-\gamma^{*}\left(j_{i}\right) \leq-\max _{m \in \mathscr{D}}\left\|\sigma\left(j_{i}\right) v\left(j_{i} \mid j_{i}\right)\right\|_{W}^{2} \\
-\left\|\left(F\left(j_{i}\right) \hat{v}\left(j_{i}\right)\right)^{*}\right\|_{R}^{2} .
\end{gathered}
$$

Apparently, $\gamma^{*}\left(j_{i}\right)$ can be as the Lyapunov function for proving the exponential stability of the closed-loop system. Then, the conclusion can be obtained.

\section{Numerical Examples}

In this section, two examples are performed to prove the effectiveness of our proposed method. The system performance will be analyzed from the simulation pictures.
5.1. Example 1. Consider the following LTI system:

$$
x(k+1)=\left[\begin{array}{ll}
1 & 0 \\
1 & 1
\end{array}\right] x(k)+\left[\begin{array}{l}
1 \\
0
\end{array}\right] u(k) .
$$

We consider the following three aspects.

Test 1 . The perfect network $\left(d=1, \delta_{g}=0\right)$. It means that there are no time delays and data quantization between sensor and controller. The sensed state $x(k)$ arrived at the controller successfully in each step $k$. Thus, we can obtain a $F(k)$ by receding horizon solving (52) in each step $k$.

Test 2. Time delay is existed in the network $\left(d=3, \delta_{g}=0\right)$. Then, we can obtain a $F(k), j_{i} \leq k<j_{i+1}$, by receding horizon solving (52) in each step $j_{i}$.

Test 3. Time delay and data quantization are coexisted in the network ( $d=3, \delta_{g}=0.3$ ). Different from Tests 1 and 2, the sensed states $x(k)$ are quantized, and then a part of the quantized $x(k)$ is transformed into the buffer.

Here, $\delta_{g}$ is the quantization parameter. We define the parameters of the performance cost as $W=I$ and $R=1$. The actual performance function is as follows:

$$
J\left(j_{i}\right)=\sum_{h=0}^{\infty}\left[x\left(j_{i+h} \mid j_{i}\right)^{T} x\left(j_{i+h} \mid j_{i}\right)+u\left(j_{i+h} \mid j_{i}\right)^{2}\right] .
$$

For Tests 1, 2, and 3, the initial conditions are $x(0)=$ $x(-1)=x(-2)=[5,5]^{T}$, and the input constraint is $|u| \leq 1$.

For the cases of Tests 1, 2, and 3, we can obtain $F$ from the first execution of the optimization process (52), respectively, as follows:

Test 1. $F=\left[\begin{array}{ll}-0.1227-0.0088\end{array}\right]$,

Test 2. $F=\left[\begin{array}{ll}-0.1227-0.0088\end{array}\right]$,

Test 3. $F=\left[\begin{array}{ll}-0.1083-0.0056\end{array}\right]$.

Through the repeated implementation of the optimization process (52), we obtain the results which are shown in Figures 4-10.

According to the simulation results in Figures 4-10, we can obtain the following conclusion.

(i) From Figures 4 and 5, we can see the steps to reach stability of system states $x_{1}, x_{2}$. The steps to reach stability of system with time delay and data quantization are more than the system with time delay and the perfect system. This is due to the deterioration of system performance with the existence of quantization. But from Figures 4 and 5, we can see that the system with time delay and data quantization can reach stability within the limited steps, which proves the effectiveness of the method in this paper.

(ii) From Figure 7, we can see that the fluctuation ranges of $u$ are increasing as the degradation of the NCS system environment. These are the changes to control move by optimization process for ensuring the stability of the system. And also it is the superiority of the MPC method. 


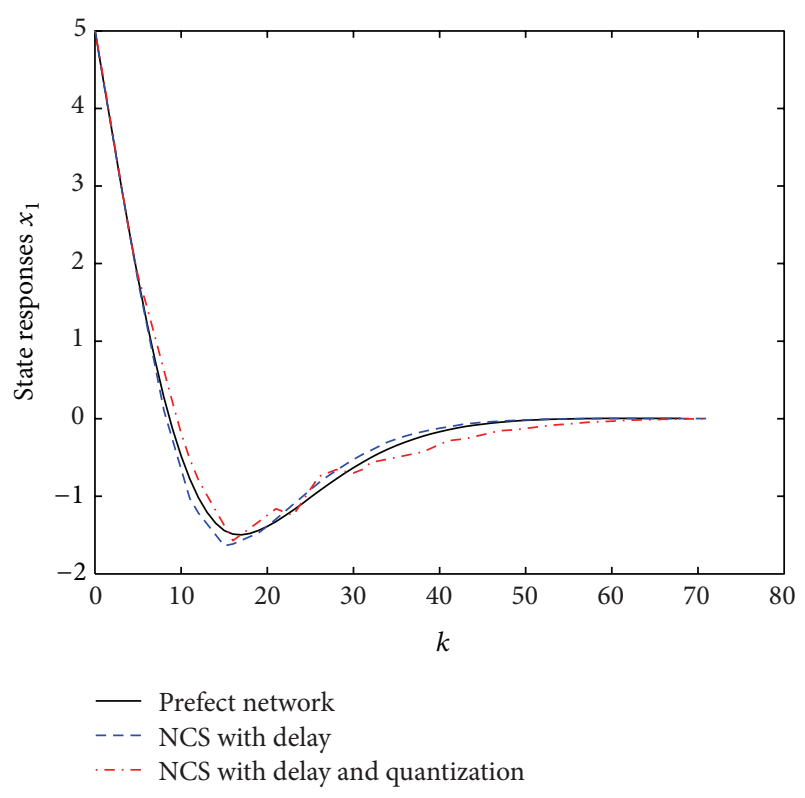

Figure 4: The state responses $x_{1}$ (Example 1).

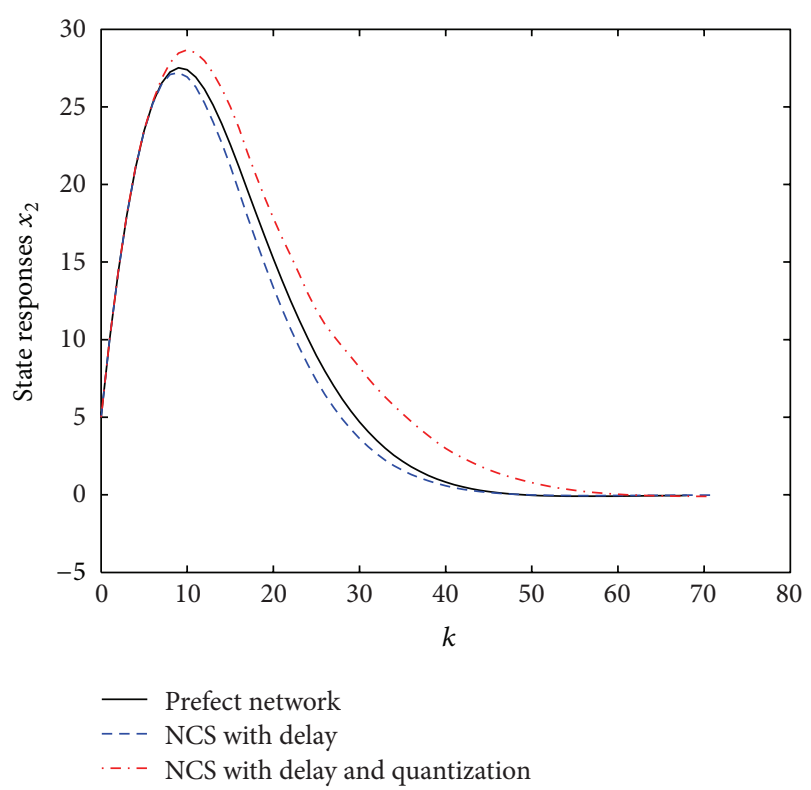

FIgURE 5: The state responses $x_{2}$ (Example 1).

(iii) In Figure 8, the evolutions of $\gamma^{*}$ are decreasing with the increase of the steps. It is shown that the closedloop system is exponentially stable. It also ensures the recursive feasibility of the proposed method.

(iv) In Figure 9, the td1, td2 mean the time delays which occur in time delay system and time delay and data quantization system separately. $\mathrm{td} 1=0$ means that there is no packet arriving at the buffer. $\operatorname{td} 1=1$ means that the current quantization state is received by the controller. td $1=2$ means that the controller receives the previous quantization state of the system. $\operatorname{td} 1=$ 3 means that $v(k-2)$ is received by the controller.

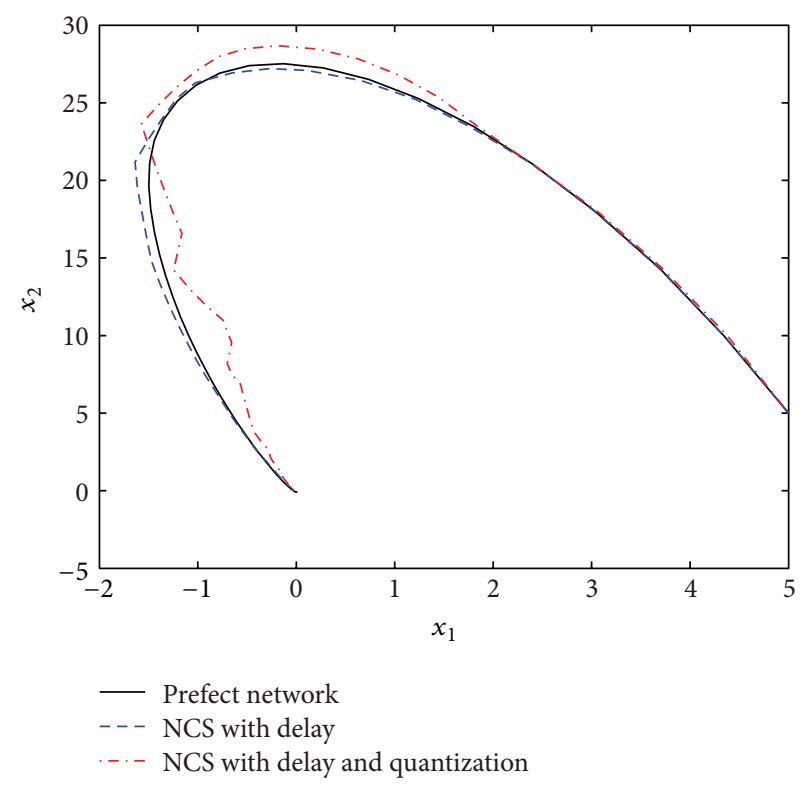

Figure 6: The state trajectory (Example 1).

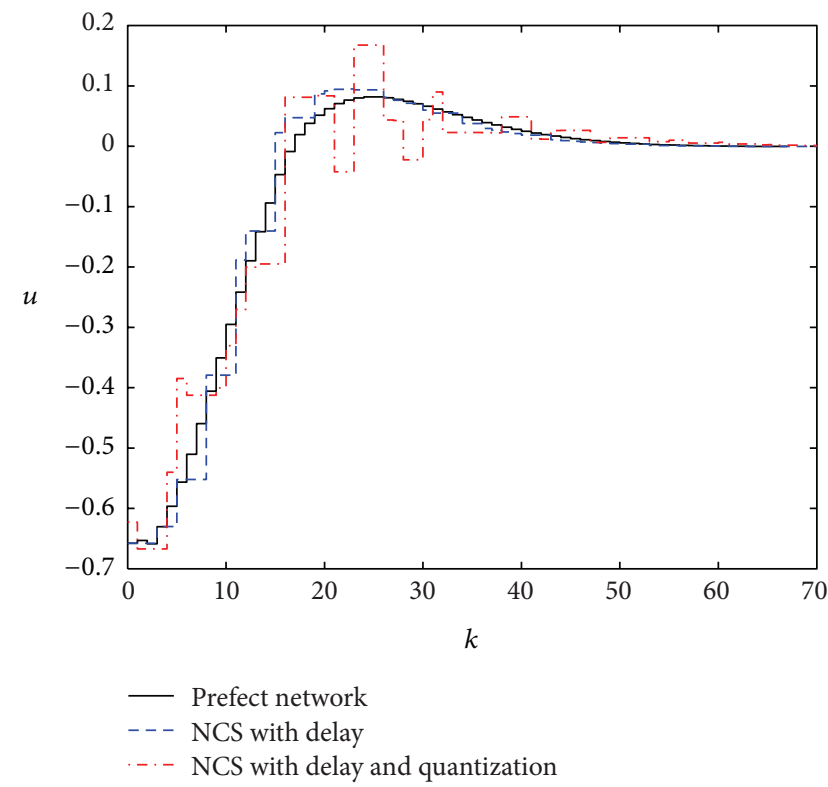

Figure 7: The control input (Example 1).

Similarly, the meaning of td 2 in Figure 10 is also the same as above.

Here, the effectiveness of the proposed method has been well validated by comparing the simulation results under different environments. It can effectively deal with the system which time delay and data quantization are coexisted.

5.2. Example 2. In this section, we consider the classical angular positioning system in $[26,27]$, which is described in Figure 11 .

The system consists of a rotating antenna which is driven by electrical motor. The direction of the antenna always points 

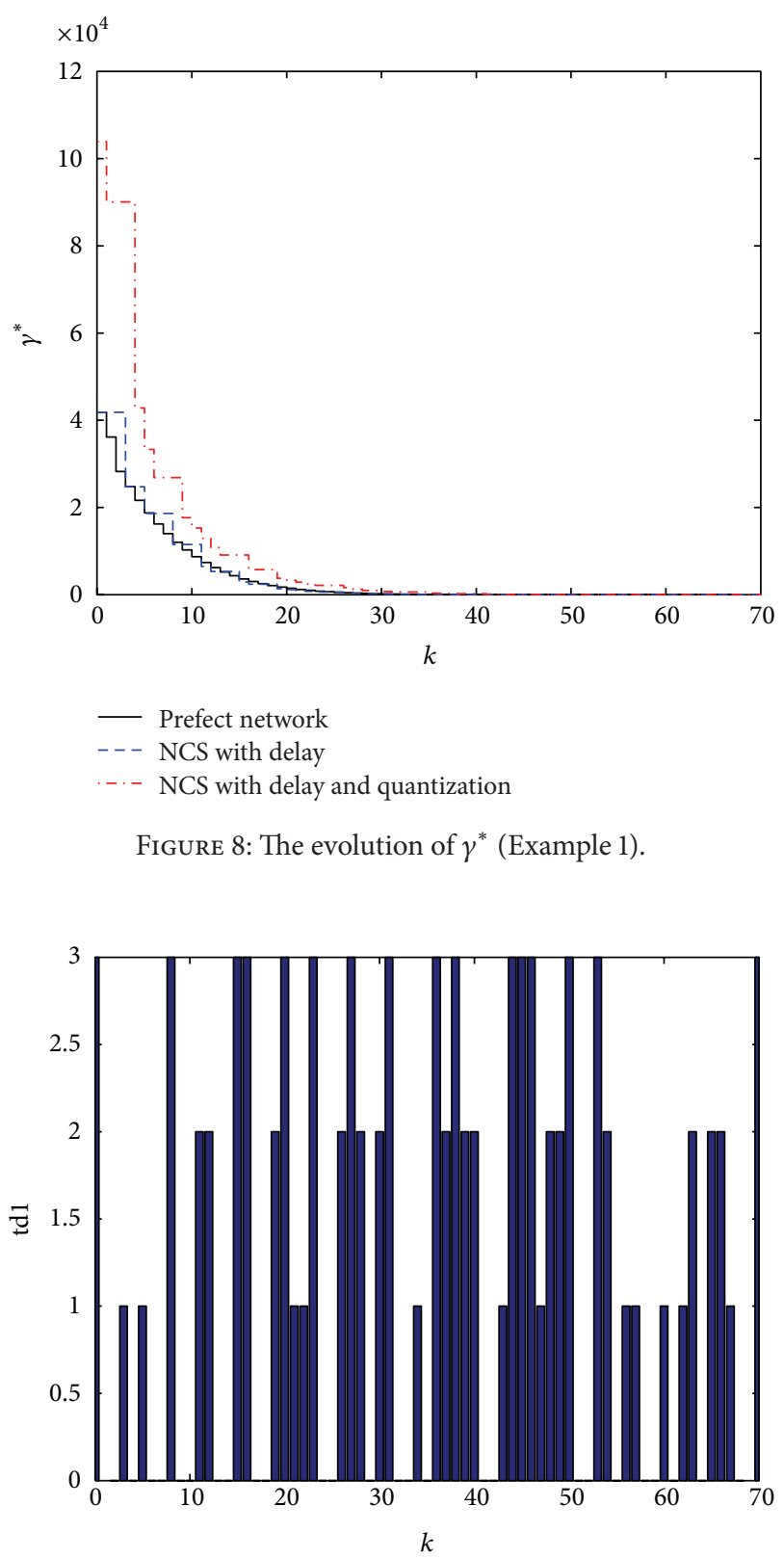

FIGURE 9: Bounded arbitrary time delays (Example 1).

to the direction of moving aircraft equipment. We assume that the angular positions of the antenna are $\theta$ (rad), angular positions of the moving object are $\theta_{r}(\mathrm{rad})$, and the angular velocity of the antenna is $\dot{\theta}\left(\mathrm{rad} \cdot \mathrm{s}^{-1}\right)$, and all the above are measurable. For details about their expressions, the readers can refer to $[26,27]$. Defining $\Delta \theta=\theta-\theta_{r}, \Delta \dot{\theta}=\dot{\theta}-\dot{\theta}_{r}$, we have

$$
\begin{aligned}
x(k+1) & =\left[\begin{array}{c}
\Delta \theta(k+1) \\
\Delta \dot{\theta}(k+1)
\end{array}\right] \\
& =\left[\begin{array}{cc}
1 & 0.1 \\
0 & 1-0.1 \alpha(k)
\end{array}\right] x(k)+\left[\begin{array}{c}
0 \\
0.1 \kappa
\end{array}\right] u(k),
\end{aligned}
$$

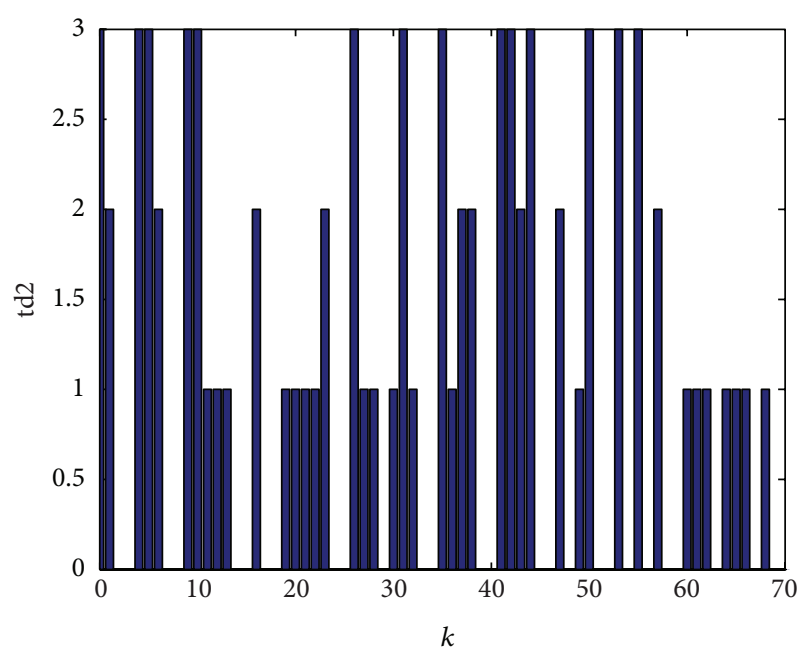

FigURE 10: Bounded arbitrary time delays (Example 1).

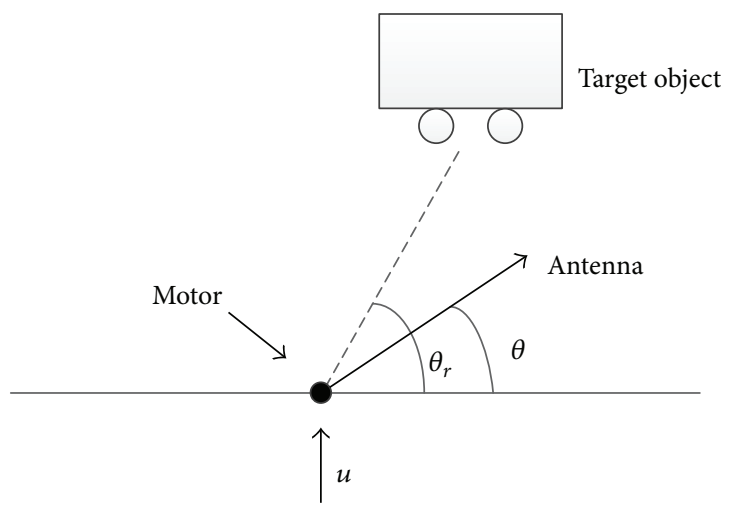

FIGURE 11: Angular positioning system.

where $\kappa=0.787 \mathrm{rad}^{-1} \mathrm{~V}^{-1} \mathrm{~s}^{-2}$ and $0.1 \mathrm{~s}^{-1} \leq \alpha(k) \leq 10 \mathrm{~s}^{-1}$. Considering $\alpha(k)=2$, the initial state $x(0)=x(-1)=$ $x(-2)=[1,0]^{T}$.

In this example, we also use the assumptions elaborated in Tests 1, 2, and 3 of Example 1. For the first execution of the optimization process (52), we can obtain $F$ with the three cases, respectively, as follows:

$$
\begin{aligned}
& \text { Test 1. } F=\left[\begin{array}{ll}
-0.1617 & -0.0780
\end{array}\right], \\
& \text { Test 2. } F=\left[\begin{array}{ll}
-0.1617 & -0.0780
\end{array}\right], \\
& \text { Test 3. } F=\left[\begin{array}{ll}
-0.1426 & -0.0692
\end{array}\right] .
\end{aligned}
$$

The simulation results are shown in Figures 12-18.

According to the simulation results in Figures 12-18, we have the similar conclusion as in Example 1.

(i) From Figures 12-14, we can see that the steps to reach stability of system states $x_{1}, x_{2}$ are almost simultaneous in three conditions. This means that the system with time delay and data quantization can obtain equally good performance as the system with time delay and the perfect system by using the method 


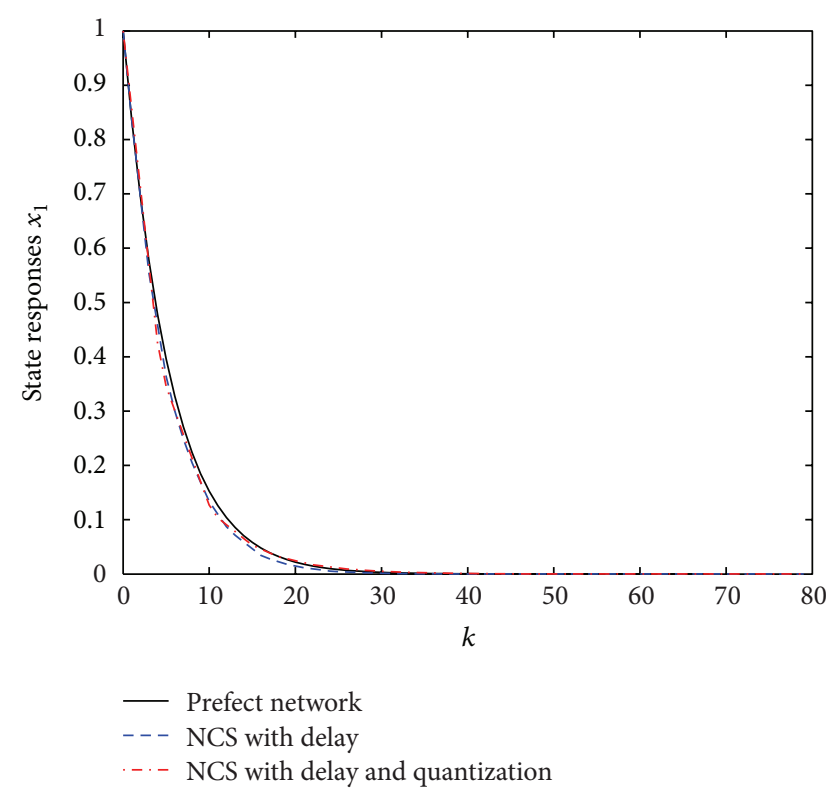

Figure 12: The state responses $x_{1}$ (Example 2).

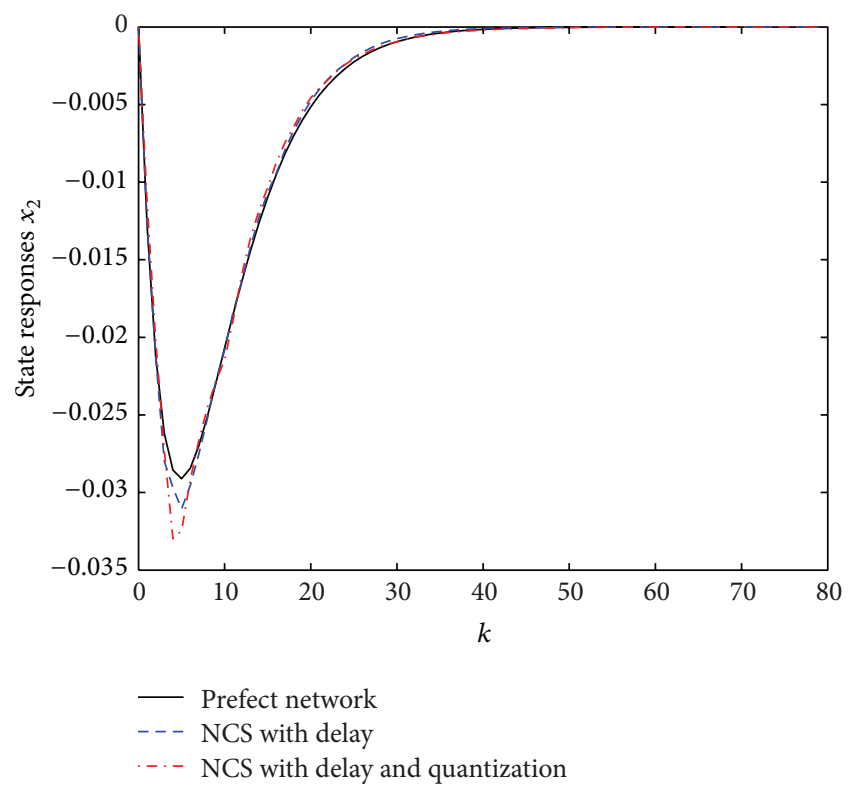

Figure 13: The state responses $x_{2}$ (Example 2).

in this paper. Figure 15 reflects the change of control move $u$ as the repeat of optimization process. The $\gamma^{*}$ in Figure 16 reflects the recursive feasibility of the method in this paper. Figures 17 and 18 show the presences of delay in the network environment.

Examples 1 and 2 reflect the applicability of the proposed method for different parameters of NCS. The two examples prove the effectiveness of the proposed method and the practical application value.

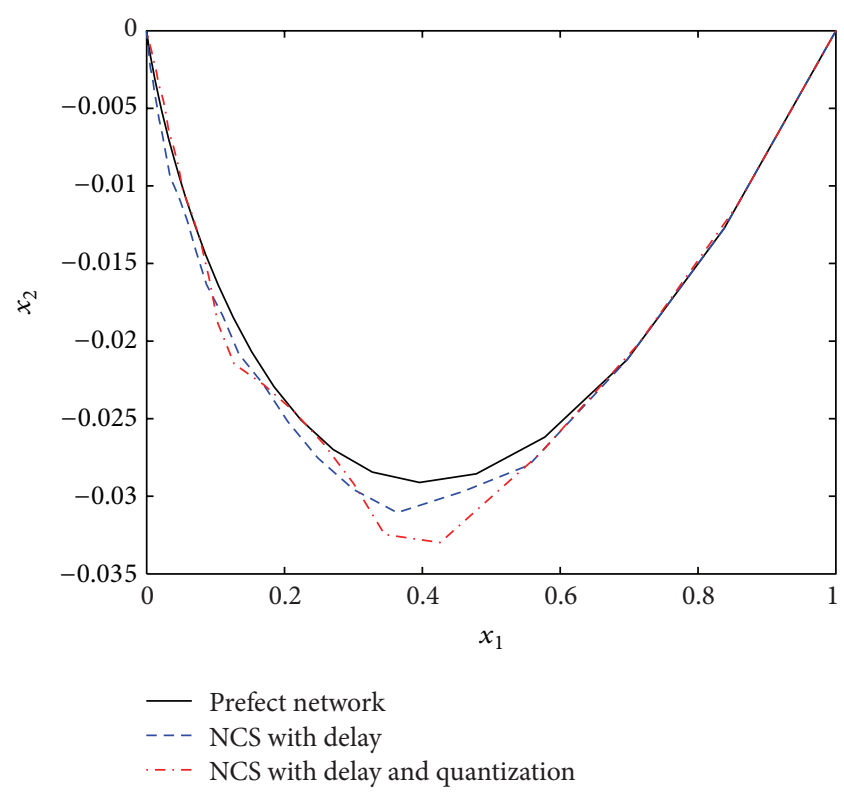

FIgure 14: The state trajectory (Example 2).

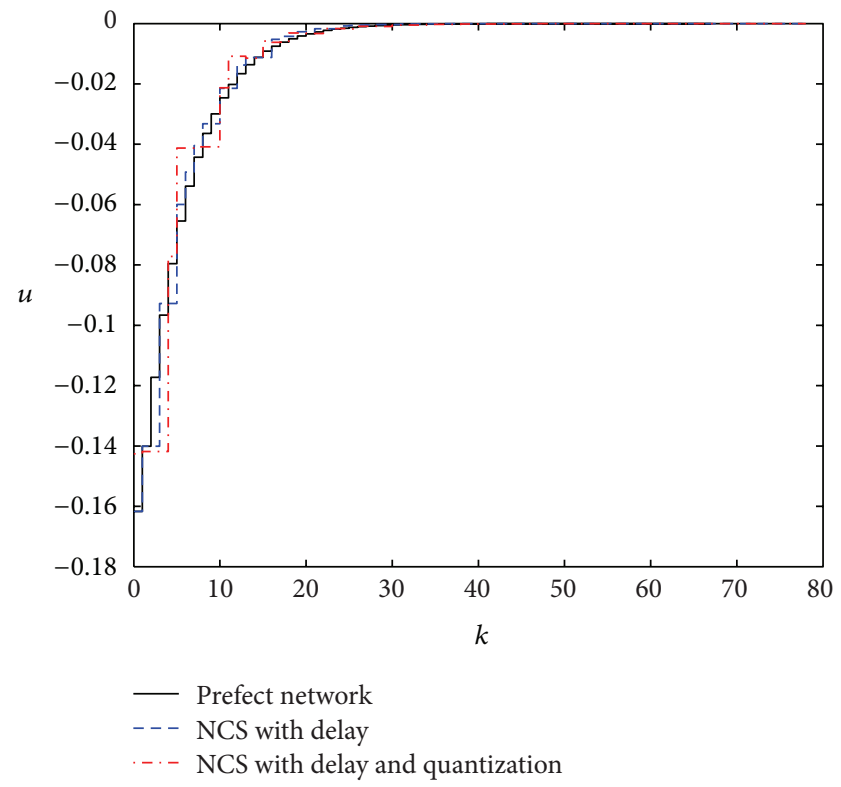

FIgURE 15: The control input (Example 2).

\section{Conclusions}

In this paper, we considered the MPC for constrained discrete time linear system under network environment. The bounded time delay and data quantization exist in NCS simultaneously. We established a novel NCS model especially for MPC method. The model predictive controller that explicitly considers the satisfaction of input and state constraints is provided. The numerical examples illustrate the effectiveness of the derived method. 


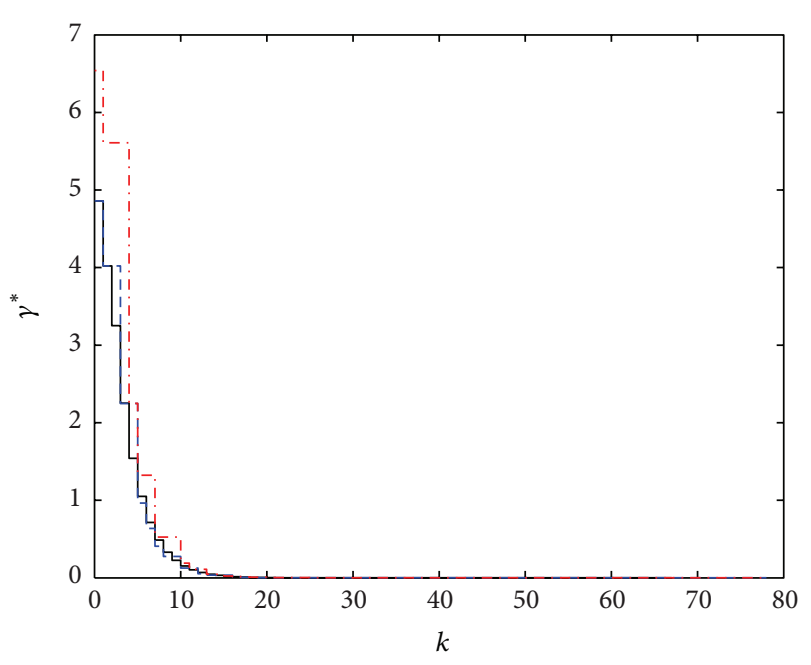

- Prefect network
--- NCS with delay
$-\cdot-$ NCS with delay and quantization

FIGURE 16: The evolution of $\gamma^{*}$ (Example 2).

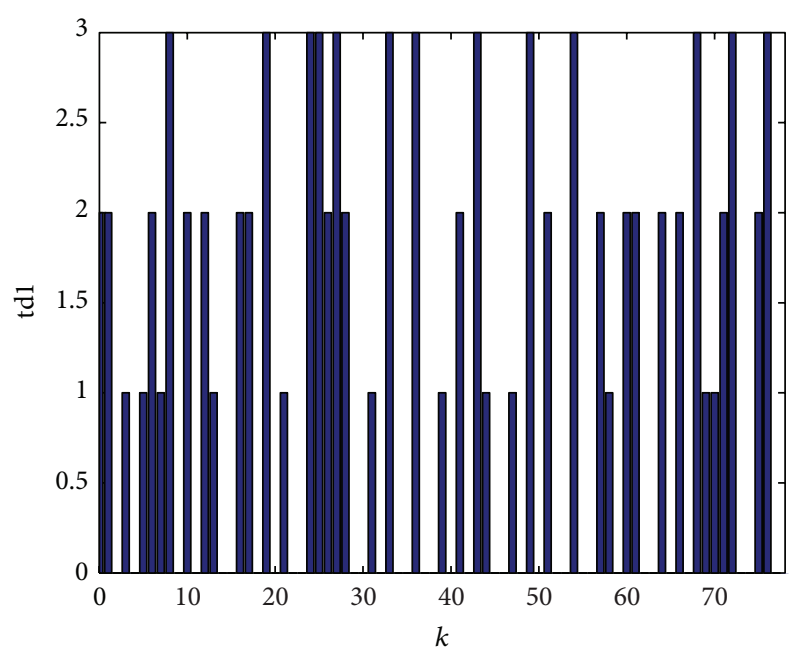

Figure 17: Bounded arbitrary time delays (Example 2).

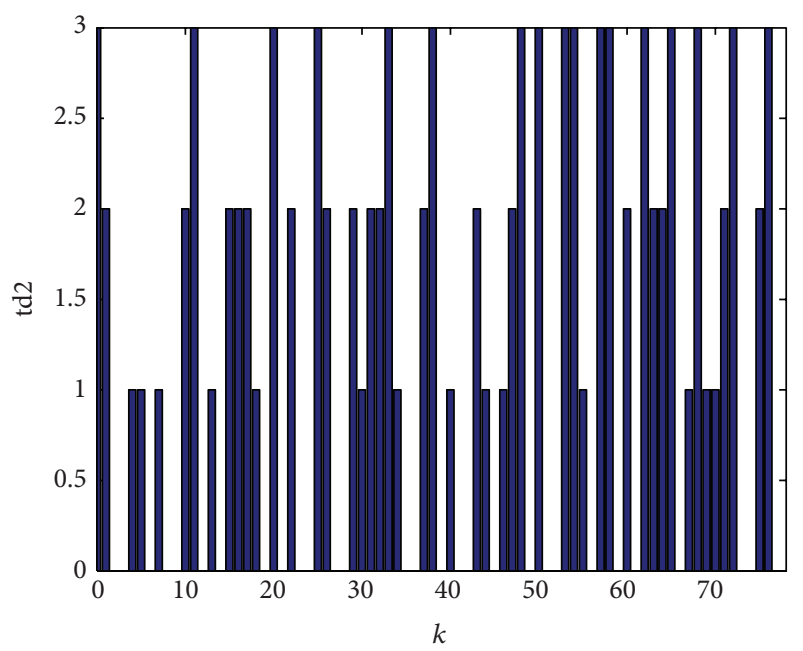

FIgURE 18: Bounded arbitrary time delays (Example 2).

\section{Conflict of Interests}

The authors declare that there is no conflict of interests regarding the publication of this paper.

\section{Acknowledgments}

This work is supported by the National Natural Science Foundation of China (61403055), the Research Project of Chongqing Science \& Technology Commission (cstc2014jcyjA40005), the Doctoral Start-Up Fund of Chongqing University of Posts and Telecommunications (A2013-14), and the Youth Natural Science Foundation of Chongqing University of Posts and Telecommunications (A2013-26).

\section{References}

[1] J. P. Hespanha, P. Naghshtabrizi, and Y. Xu, "A survey of recent results in networked control systems," Proceedings of the IEEE, vol. 95, no. 1, pp. 138-172, 2007.

[2] L. Qiu, F. Q. Yao, and X. P. Zhong, "Stability analysis of networked control systems with random time delays and packet dropouts modeled by markov chains," Journal of Applied Mathematics, vol. 2013, Article ID 715072, 10 pages, 2013.

[3] G.-S. Tian, F. Xia, and Y.-C. Tian, "Predictive compensation for variable network delays and packet losses in networked control systems," Computers and Chemical Engineering, vol. 39, pp. 152162,2012

[4] J. Nilsson, B. Bernhardsson, and B. Wittenmark, "Stochastic analysis and control of real-time systems with random time delays," Automatica, vol. 34, no. 1, pp. 57-64, 1998.

[5] S. S. Hu and Q. X. Zhu, "Stochastic optimal control and analysis of stability of networked control systems with long delay," Automatica, vol. 39, no. 11, pp. 1877-1884, 2003.

[6] J. L. Xiong and J. Lam, "Stabilization of discrete-time Markovian jump linear systems via time-delayed controllers," Automatica, vol. 42, no. 5, pp. 747-753, 2006.

[7] G. C. Goodwin, H. Haimovich, D. E. Quevedo, and J. S. Welsh, "A moving horizon approach to networked control system design," IEEE Transactions on Automatic Control, vol. 49, no. 9, pp. 1427-1445, 2004.

[8] J. Wu, L. Zhang, and T. Chen, "Model predictive control for networked control systems," International Journal of Robust and Nonlinear Control, vol. 19, no. 9, pp. 1016-1035, 2009.

[9] X. L. Wang, C. G. Fei, and Z. Z. Han, "Adaptive predictive functional control for networked control systems with random delays," International Journal of Automation and Computing, vol. 8, no. 1, pp. 62-68, 2011.

[10] X.-M. Tang and B.-C. Ding, "Design of networked control systems with bounded arbitrary time delays," International Journal of Automation and Computing, vol. 9, no. 2, pp. 182-190, 2012.

[11] N. Elia and S. K. Mitter, "Stabilization of linear systems with limited information," IEEE Transactions on Automatic Control, vol. 46, no. 9, pp. 1384-1400, 2001.

[12] M. Fu and L. Xie, "The sector bound approach to quantized feedback control," IEEE Transactions on Automatic Control, vol. 50, no. 11, pp. 1698-1711, 2005.

[13] W. Pan, H. M. Jiang, and C. Q. Zhu, "Stable region analysis of networked control systems with uniform quantization," in 
Proceedings of the 26th Chinese Control Conference (CCC '07), pp. 80-83, July 2007.

[14] D. Nesic, "A unified approach to analysis and design of networked and quantized control systems," in Proceedings of the Chinese Control and Decision Conference, pp. 43-52, Yantai, China, July 2008.

[15] Y. W. Zhang and Q. F. Meng, "Stability of networked control system with quantization," in Proceedings of the Chinese Control and Decision Conference (CCDC '09), pp. 559-564, Guilin, China, June 2009.

[16] X. M. Tang and B. C. Ding, "Model predictive control of linear systems over networks with data quantizations and packet losses," Automatica, vol. 49, no. 5, pp. 1333-1339, 2013.

[17] Y. G. Xi, D. W. Li, and S. Lin, "Model predictive control-status and challenges," Acta Automatica Sinica, vol. 39, no. 3, pp. 222236, 2013.

[18] A. D. Liu, L. Yu, W. A. Zhang, and D. F. He, "Model predictive control for networked control systems with random delay and packet disordering," in Proceedings of the 8th Asian Control Conference (ASCC '11), pp. 653-658, May 2011.

[19] P. Varutti, B. Kern, T. Faulwasser, and R. Findeisen, "Eventbased model predictive control for networked control systems," in Proceedings of the 48th IEEE Conference on Decision and Control held jointly with 28th Chinese Control Conference (CDC/CCC '09), pp. 567-572, December 2009.

[20] B. Xue, N. Li, S. Li, and Q. Zhu, "Robust model predictive control for networked control systems with quantisation," IET Control Theory \& Applications, vol. 4, no. 12, pp. 2896-2906, 2010.

[21] B. C. Ding, "Stabilization of linear systems over networks with bounded packet loss and its use in model predictive control," Automatica, vol. 47, no. 11, pp. 2526-2533, 2011.

[22] M. V. Kothare, V. Balakrishnan, and M. Morari, "Robust constrained model predictive control using linear matrix inequalities," in Proceedings of the American Control Conference, pp. 440-444, July 1994.

[23] Y. Lu and Y. Arkun, "Quasi-min-max MPC algorithms for LPV systems," Automatica, vol. 36, no. 4, pp. 527-540, 2000.

[24] B. C. Ding and L. H. Xie, "Stabilization of linear systems over networks with double-sided, finite bounded, arbitrary packet loss," in Proceedings of the 7th IEEE International Conference on Control and Automation (ICCA '09), pp. 920-925, Christchurch, New Zealand, December 2009.

[25] B. Ding and X. Tang, "Designing model predictive control with one free control move for linear systems over networks with bounded packet loss," in Proceedings of the 29th Chinese Control Conference (CCC '10), pp. 3134-3139, Beijing, China, July 2010.

[26] M. V. Kothare, V. Balakrishnan, and M. Morari, "Robust constrained model predictive control using linear matrix inequalities," Automatica, vol. 32, no. 10, pp. 1361-1379, 1996.

[27] H. Kwakernaak, R. Sivan, and M. Morari, Linear Optimal Control Systems, Wiley-Interscience, New York, NY, USA, 1972. 

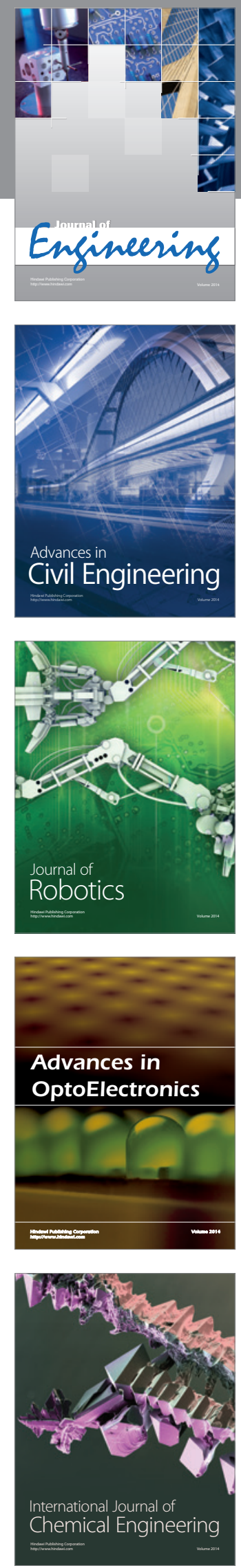

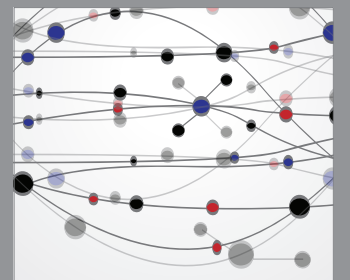

The Scientific World Journal
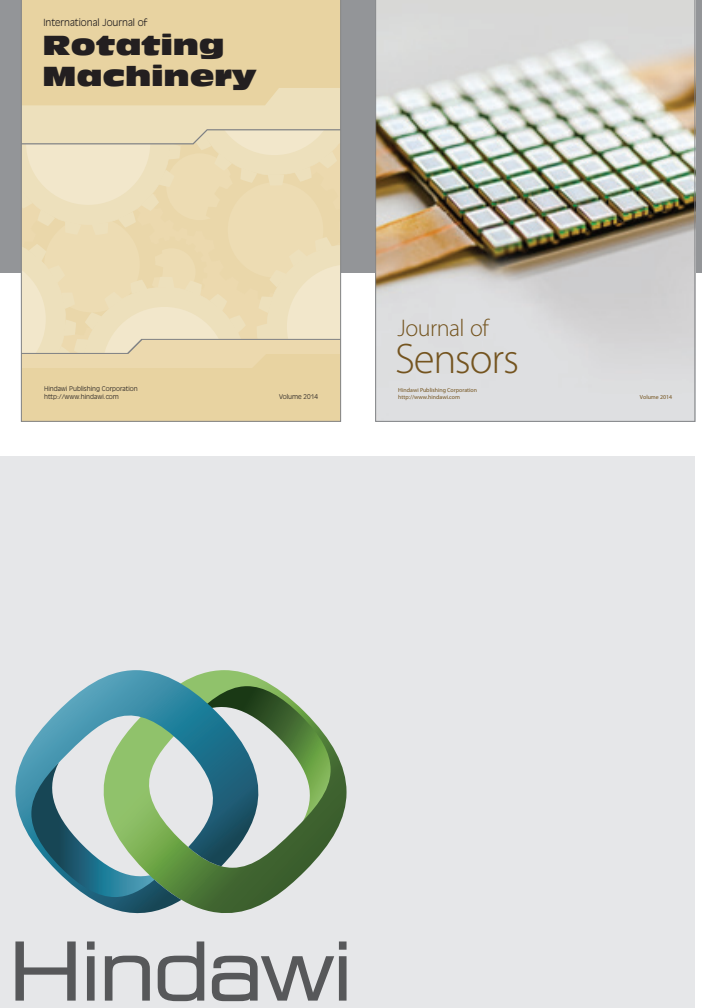

Submit your manuscripts at http://www.hindawi.com
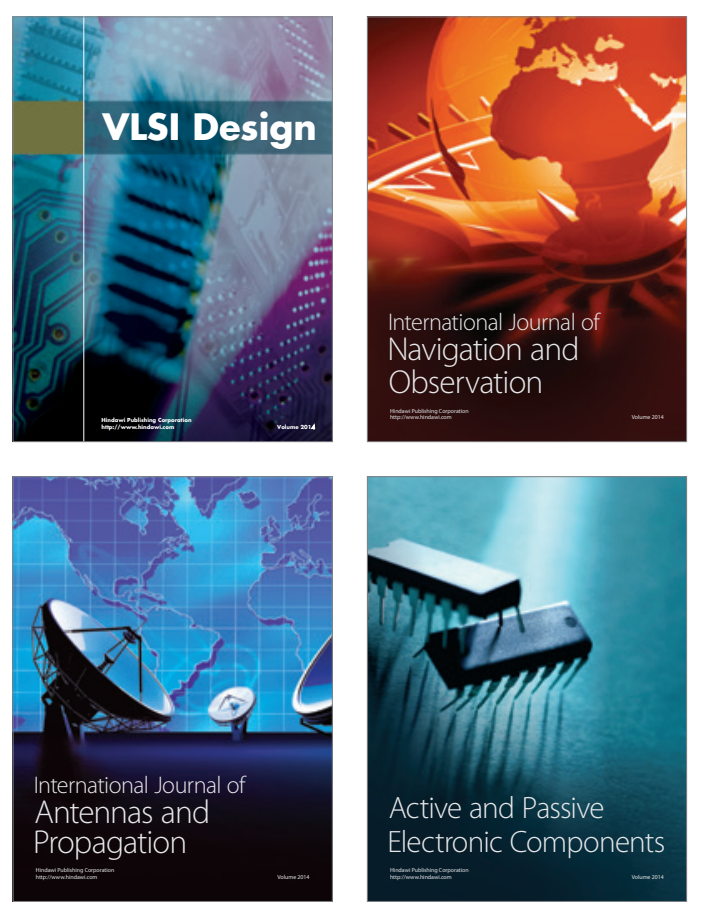
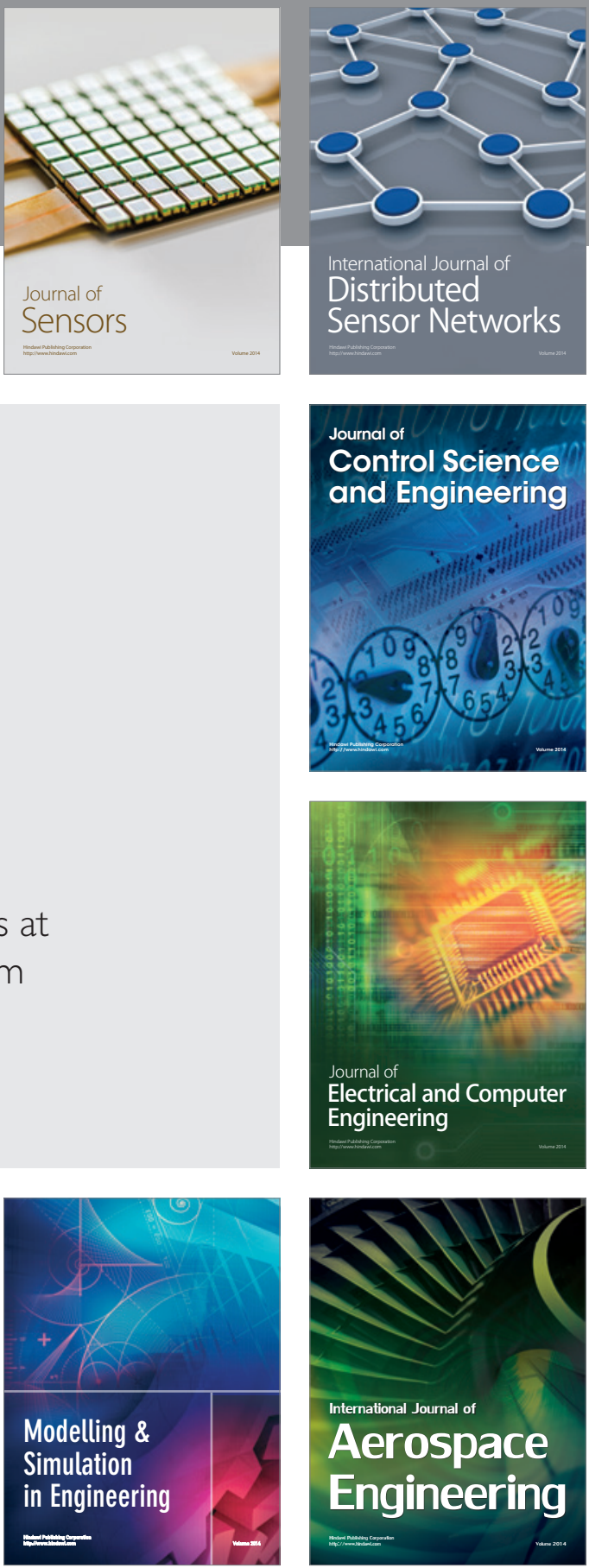

Journal of

Control Science

and Engineering
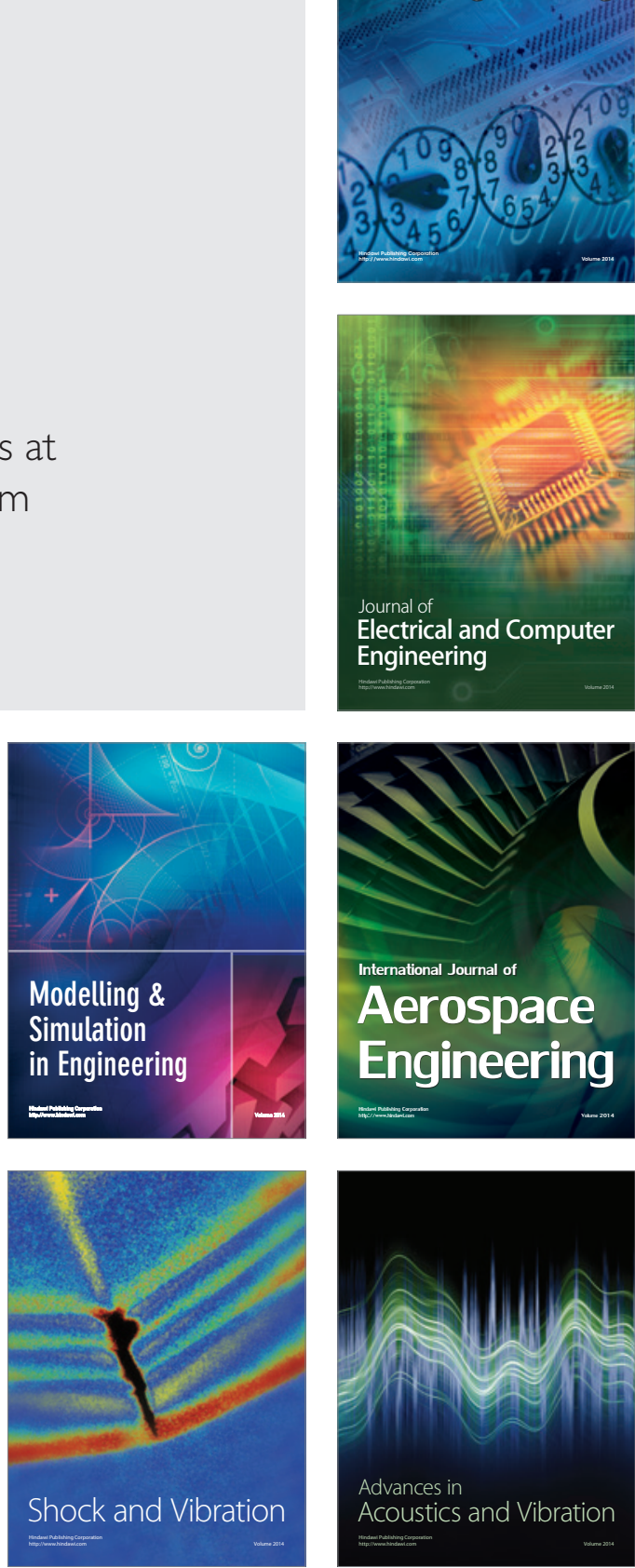